\title{
Successive-Relaying-Aided Decode-and-Forward Coherent Versus Noncoherent Cooperative Multicarrier Space-Time Shift Keying
}

\author{
Mohammad Ismat Kadir, Li Li, Sheng Chen, Fellow, IEEE, and Lajos Hanzo, Fellow, IEEE
}

\begin{abstract}
Successive-relaying-aided (SR) cooperative multicarrier (MC) space-time shift keying (STSK) is proposed for frequency-selective channels. We invoke $S R$ to mitigate the typical $50 \%$ throughput loss of conventional half-duplex relaying schemes and MC code-division multiple access (MC-CDMA) to circumvent the dispersive effects of wireless channels and to reduce the SR-induced interference. The distributed relay terminals form two virtual antenna arrays (VAAs), and the source node (SN) successively transmits frequency-domain (FD) spread signals to one of the VAAs, in addition to directly transmitting to the destination node (DN). The constituent relay nodes (RNs) of each VAA activate cyclic-redundancy-checking-based (CRC) selective decode-and-forward (DF) relaying. The DN can jointly detect the signals received via the SN-to-DN and VAA-to-DN links using a low-complexity single-stream-based joint maximum-likelihood (ML) detector. We also propose a differentially encoded cooperative MC-CDMA STSK scheme to facilitate communications over hostile dispersive channels without requiring channel estimation (CE). Dispensing with $\mathrm{CE}$ is important since the relays cannot be expected to altruistically estimate the SN-to-RN links for simply supporting the source. Furthermore, we propose softdecision-aided serially concatenated recursive systematic convolutional (RSC) and unity-rate-coded (URC) cooperative MC STSK and investigate its performance in both coherent and noncoherent scenarios.
\end{abstract}

Index Terms-Coherent and noncoherent detection, decodeand-forward (DF), frequency-selective channel, multicarrier code-division multiple access (MC-CDMA), space-time shift keying (STSK), successive relaying (SR).

\section{INTRODUCTION}

$\mathbf{T}$ HE concept of space-time shift keying (STSK) [1]-[3] was recently developed, which drew its motivation from the extremely simple architecture of spatial modulation (SM) [4] and space shift keying (SSK) [4]. STSK complemented the simplicity of SM and SSK by the rate versus diversity tradeoffs provided by linear dispersion codes (LDCs) [5], [6]. LDCs constitute a generic family subsuming both space-time block codes

Manuscript received April 23, 2012; revised January 28, 2013; accepted February 4, 2013. Date of publication February 12, 2013; date of current version July 10, 2013. This work was supported in part by the Commonwealth Scholarship Commission in the U.K., by the Research Councils U.K. under the auspices of the India-U.K. Advanced Technology Centre, by the European Union under the Concerto Project, and by the European Research Council under the Advanced Fellow Grant. The review of this paper was coordinated by Prof. W. A. Hamouda.

The authors are with the School of Electronics and Computer Science, University of Southampton SO17 1BJ, U.K. (e-mail: mik1g09@ecs.soton.ac.uk; 115e08@ecs.soton.ac.uk; sqc@ecs.soton.ac.uk; 1h@ecs.soton.ac.uk).

Color versions of one or more of the figures in this paper are available online at http://ieeexplore.ieee.org.

Digital Object Identifier 10.1109/TVT.2013.2246800
[7] and the Bell Laboratories layered space-time (BLAST) [8] and vertical-BLAST (V-BLAST) [9], [10] schemes and are capable of outperforming both, albeit at the cost of higher decoding complexity. On the other hand, SM and SSK simply activate only one of the transmit antenna elements, hence resulting in a low-complexity detector. Against this background, STSK was designed to activate a single one from $Q$ dispersion matrices (DMs) along with the conventional phase-shift keying (PSK) or quadrature amplitude modulation (QAM) symbols. Thus, STSK is capable of attaining the benefits of LDCs while relying on a low-complexity design and decoding principle [11].

On the other hand, the concept of cooperative space-time processing [12], [13] has also become popular in recent years, owing to its benefits accruing from the geographically distributed nature of relay nodes (RNs), where the relays may be viewed as the distributed elements of a multiple-inputmultiple-output (MIMO) system, with each element experiencing uncorrelated fading. Recently, the concept of cooperative STSK [14] has been proposed for frequency-flat Rayleigh fading channels to benefit from cooperation, although naturally, this scheme suffers from the usual throughput loss imposed by the relaying strategy employed. The introduction of successive relaying (SR) [15], on the other hand, is potentially capable of recovering the half-duplex multiplexing loss; hence, it was successfully used in [16] as a near-capacity cooperative space-time coding architecture. Furthermore, a noncoherentdetection-based scheme employing both multiple-symbol differential sphere decoding and SR was conceived in [17] and [18]. However, the SR regime imposes additional interference both at the RNs and at the destination nodes (DNs) [15], namely, the interrelay interference (IRI) and the cochannel interference (CCI), which limit its performance. A differentialSTSK-aided (DSTSK) successive-relay-assisted decode-andforward (DF) scheme was proposed for cooperative multiuser code-division multiple-access (CDMA) systems [19], which mitigates the throughput loss imposed by half-duplex relaying. However, this scheme is applicable only to the nondispersive MIMO system.

To exploit the diversity benefits of cooperative schemes and to circumvent the channel-induced dispersion while mitigating the throughput loss imposed by half-duplex relaying, we propose a novel SR-based DF cooperative multicarrier (MC) STSK scheme. The novel contributions of this paper are as follows.

1) We intrinsically amalgamate for the first time $M C$ transmissions with a cooperative STSK system to 
communicate reliably over hostile multipath channels. More particularly, we propose MC-CDMA-based cooperative STSK for achieving an improved diversity gain to recover the original input sequence. Although orthogonal frequency-division multiplexing/multiple access (OFDM/OFDMA) or single-carrier frequency-division multiple access (SC-FDMA) can be employed for mitigating the channel-induced dispersion in our STSK-based system [20], [21], MC-CDMA is capable of providing the additional benefit of frequency-domain (FD) diversity. The incorporation of MC-CDMA has the further benefit of substantially reducing both the IRI and CCI, when employing the specific SR regime of [17].

2) We propose a SR-aided cyclic-redundancy-checkingbased (CRC) selective DF cooperative STSK scheme. The SR invoked in this context helps to recover the multiplexing loss of conventional half-duplex relaying schemes.

3) We also propose a new modality for the joint detection [14], [17] of the FD-despread signals gleaned from two successively arriving frames at the $\mathrm{DN}$ via the source node (SN)-to-DN and virtual antenna array (VAA)-to-DN links by using the single-stream-based maximum-likelihood (ML) detector of [22]. The joint detector takes advantage of the interstream interferencefree nature of STSK schemes since always a single DM is activated.

4) We demonstrate that the coherent SR-aided MC-CDMA STSK scheme performs well, but it might be unrealistic to expect that the RNs altruistically estimate the SN-toRN channels. As a potential remedy, a new noncoherent cooperative MC STSK arrangement using unitary DMs, rather than using the nonlinear Cayley transform [2], [23], is proposed.

5) We propose a powerful serially concatenated turboprinciple-based channel-coded cooperative MC scheme, where the DN iteratively exchanges soft information between the component decoders before finally outputting the estimated source information. The performance of the scheme is evaluated both in the context of the coherent and differential schemes and compared against the corresponding maximum achievable capacity benchmark, using our extrinsic-information-transfer (EXIT)chart-based semi-analytical method.

The remainder of this paper is organized as follows. In Section II, we present an overview of the proposed system. The joint detection of the signals arriving from the SN-DN and VAA-DN links is discussed in Section III. The proposed differential MC cooperative STSK and the channel-coded softdecision-based MC cooperative STSK schemes are outlined in Sections IV and V, respectively. In Section VI, the performance of the proposed scheme is investigated. Finally, we conclude the paper in Section VII.

\section{System Overview of The Coherent Scheme}

The typical four-node network topology and transmission protocol of the classic SR scheme [15] is portrayed in Fig. 1,

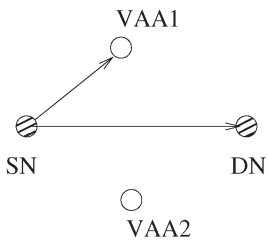

(a)

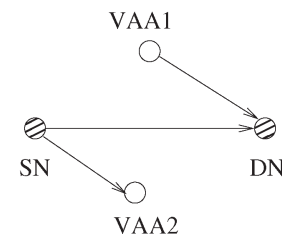

(b)

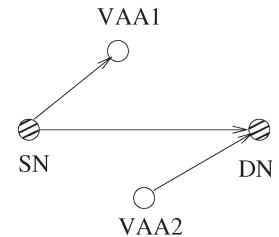

(c)
Fig. 1. Transmission protocol of SR-aided cooperation during different time slots.

whereas the overall system architecture of our proposed scheme is depicted in Fig. 2, where the SN, DN, and the two VAAs taking part in SR are explicitly labeled. Additionally, for the sake of enabling the CRC at the RNs, frame-based rather than symbol-based transmissions are adopted. We assume that the distances between the different RNs of the same VAA are negligible with respect to the distance between the SN and the DN (or between the SN and the VAA). Accordingly, a VAA is assumed to exhibit a unitary nature, when considering the geometric relationship among $\mathrm{SN} s$, the $i$ th VAA $v_{i}$, and $\mathrm{DN} d$. The average path-loss gains of the SN-VAA and VAA-DN links with respect to the SN-DN links are denoted by $G_{s v_{i}}=\left(D_{s d} / D_{s v_{i}}\right)^{\alpha}, i=1,2$, and $G_{v_{i} d}=\left(D_{s d} / D_{v_{i} d}\right)^{\alpha}$, $i=1,2$, respectively, where $\alpha$ is the path-loss exponent, and $D_{a a^{\prime}}, a, a^{\prime} \in\left\{s, v_{i}, d\right\}$ represents the distance between nodes $a$ and $a^{\prime}$. Furthermore, we assume a symmetric structure, where $D_{s v_{1}}, D_{v_{1} d}, G_{s v_{1}}$, and $G_{v_{1} d}$ are identical to $D_{s v_{2}}, D_{v_{2} d}, G_{s v_{2}}$, and $G_{v_{2} d}$, respectively. Furthermore, all the possible channel paths are assumed to be frequency-selective Rayleigh fading channels.

\section{A. SN's Transmission Model}

The SN first attaches the CRC bits to its information bits and transmits them both to the $M$ RNs of a VAA and to the DN in each of its broadcast phases, as shown in Fig. 2. To be specific, the CRC-protected bits are first mapped to the $\mathcal{L}^{\prime}$ PSK or $\mathcal{L}^{\prime}$-QAM symbol blocks [14] according to $\boldsymbol{S}_{s}(k) \triangleq$ $\left[s_{1}(k), \ldots, s_{b}(k)\right]^{T} \in \mathbb{C}^{b \times 1}$, where $k(1,2, \ldots)$ represents the block index and each block carries $\left(b \cdot \log _{2} \mathcal{L}^{\prime}\right)$ bits. Let us also define the frame length $L_{f}$ as the number of $\left(b \cdot \log _{2} \mathcal{L}^{\prime}\right)$ bit signal blocks transmitted in each frame; hence, the block index $k$ is related to the $l_{f}$ th block of the $n$th frame by $k=$ $\left(n \times L_{f}+l_{f}\right)$.

We divide all the frames into two sets. The frame being broadcast when VAA1 of Fig. 1 is receiving is referred to as frame-A, which is spread by the spreading sequence $C_{A}^{u}$ for user $u,(u=1,2, \ldots, U)$. By contrast, the frame being broadcast when VAA2 is receiving is referred to as frame-B, which is spread by $C_{B}^{u}$, where both $C_{A}^{u}=\left[C_{A}^{u}(1), C_{A}^{u}(2)\right.$, $\left.\ldots, C_{A}^{u}\left(S_{f}\right)\right]$ and $C_{B}^{u}=\left[C_{B}^{u}(1), C_{B}^{u}(2), \ldots, C_{B}^{u}\left(S_{f}\right)\right]$ have a spreading factor of $S_{f}$. Both the spreading sequences $C_{A}^{u}$ and $C_{B}^{u}$ are $S_{f}$-length vectors whose chips are denoted by $C_{A}^{u}\left(s_{f}\right)$, $s_{f}=1,2, \ldots, S_{f}$, and $C_{B}^{u}\left(s_{f}\right), s_{f}=1,2, \ldots, S_{f}$, respectively. The block index $k(1,2, \ldots)$ of the signal block $\boldsymbol{S}_{s}(k)$ is related to the index $k^{\prime}(1,2, \ldots)$ of the spread blocks, e.g., $\boldsymbol{S}_{c}\left(k^{\prime}\right)=\left[C_{A}^{u}\left(s_{f}\right) \boldsymbol{S}_{s}(k)\right] \in \mathbb{C}^{b \times 1}$ by $k^{\prime}=k \times S_{f}+s_{f} s_{f}=$ 


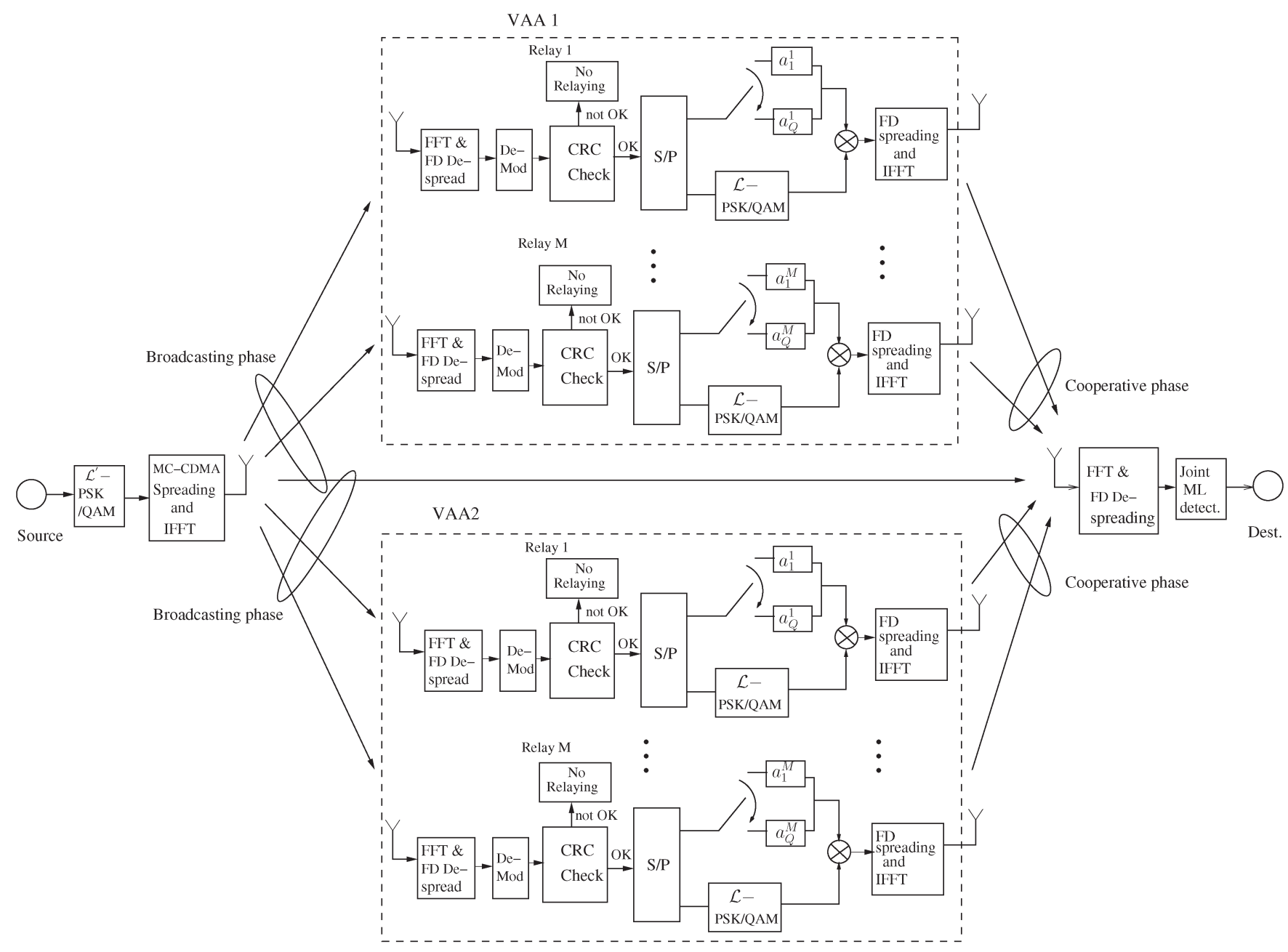

Fig. 2. Transmission model of SR-aided STSK employing FD-spreading/despreading and IFFT/FFT-based MC-CDMA modem. Each of the two VAAs consists of $M$ number of RNs, which activate relaying depending on the outcome of CRC. The chip-waveform-based spread-despread paradigm overcomes SR induced interference, whereas the scheme is benefitted from the joint single-stream-based ML detector.

$1,2, \ldots, S_{f}$, whereas the spread blocks are generated using the spreading sequence $C_{A}^{u}\left(s_{f}\right)$ or $C_{B}^{u}\left(s_{f}\right)$, depending on whether frame-A or frame-B is being transmitted. We assume that a particular spread block is transmitted over $b$ time intervals and the fading envelope during the transmission of a block of $b$ symbols remains constant. For the sake of readability, we omit the user index $u$ in the following, except in (30) and in (31), shown below where the multiuser scenario is specifically considered. The different users are separated by their mutually orthogonal user-specific spreading sequences, albeit the multiuser scenario is not explicitly shown in Fig. 2 for avoiding obfuscation. Assuming the spread frame length $\left(L_{f} \times S_{f}\right)$ to be a multiple of the number $N_{c}$ of subcarriers, whereas $N_{c}$ is equal to or a multiple of $S_{f}$, each frame is mapped to the $N_{c}$ subcarriers using the $N_{c}$-point inverse discrete Fourier transform (DFT). Then, the cyclic prefixes (CPs), which are designed to be longer than the channel's delay spread, are attached to avoid any intersymbol interference (ISI). The linear convolution between the time-domain (TD) channel input signals and the channel impulse response (CIR) is transformed into scalar multiplication in the FD [24]. Hence, the FD signals $\boldsymbol{Y}_{s d}^{A}\left(k^{\prime}\right) \in \mathbb{C}^{b \times 1}$ and $\boldsymbol{Y}_{s d}^{B}\left(k^{\prime}\right) \in \mathbb{C}^{b \times 1}$ received at the DN from the direct SN-DN link of a particular user and $\boldsymbol{Y}_{s v_{i}}^{m}\left(k^{\prime}\right) \in \mathbb{C}^{b \times 1}, i=1,2$ at the $m$ th $\mathrm{RN}$ of each VAA are given, after $\mathrm{CP}$ removal and DFT, by

$$
\begin{gathered}
\boldsymbol{Y}_{s v_{1}}^{m}\left(k^{\prime}\right)=\sqrt{G_{s v_{1}}} \tilde{h}_{s v_{1}}^{m}\left(k^{\prime}\right)\left[C_{A}\left(s_{f}\right) \boldsymbol{S}_{s}(k)\right]+\tilde{\boldsymbol{N}}_{v_{1}}^{m}\left(k^{\prime}\right) \\
(\text { Frame-A }) \\
\boldsymbol{Y}_{s v_{2}}^{m}\left(k^{\prime}\right)=\sqrt{G_{s v_{2}}} \tilde{h}_{s v_{2}}^{m}\left(k^{\prime}\right)\left[C_{B}\left(s_{f}\right) \boldsymbol{S}_{s}(k)\right]+\tilde{\boldsymbol{N}}_{v_{2}}^{m}\left(k^{\prime}\right) \\
\quad(\text { Frame-B }) \\
\boldsymbol{Y}_{s d}^{A}\left(k^{\prime}\right)=\tilde{h}_{s d}\left(k^{\prime}\right)\left[C_{A}\left(s_{f}\right) \boldsymbol{S}_{s}(k)\right]+\tilde{\boldsymbol{N}}_{d}\left(k^{\prime}\right) \\
\quad(\text { Frame-A) } \\
\boldsymbol{Y}_{s d}^{B}\left(k^{\prime}\right)=\tilde{h}_{s d}\left(k^{\prime}\right)\left[C_{B}\left(s_{f}\right) \boldsymbol{S}_{s}(k)\right]+\tilde{\boldsymbol{N}}_{d}\left(k^{\prime}\right)
\end{gathered}
$$

(Frame-B)

where $\tilde{h}_{s v_{i}}^{m}$ and $\tilde{h}_{s d}$ denote the FD channnel coefficients between the SN and the $m$ th RNs of VAA $i$ and between the $\mathrm{SN}$ and the DN, respectively, obeying the complex-valued Gaussian distributions of $\mathcal{C N}\left(0, \sigma_{s v_{i}}^{2}\right)$ and $\mathcal{C N}\left(0, \sigma_{s d}^{2}\right)$, respectively. Each component of the noise vectors $\tilde{N}_{v_{i}}^{m}$ and $\tilde{N}_{d}$ in (1)-(4) is a complex-valued Gaussian variable of $\mathcal{C N}\left(0, N_{0}\right)$, with $N_{0}$ representing the noise variance. 


\section{B. $V A A$}

As aforementioned, each of the two VAAs taking part in the SR paradigm is composed of $M$ RNs and operates on the principle of the CRC-enabled selective DF strategy of [14] and [25]. The signal received at each RN of a VAA is decoded following FD MC-CDMA despreading. For a scenario supporting multiple users, the source information on different users are jointly detected by a ML multiuser detector (ML-MUD), as discussed in [26]. If the signal at any $\mathrm{RN}$ of the VAA is deemed to be correctly decoded by the CRC, then that specific $\mathrm{RN}$ is allowed to engage in relaying. The same $\mathrm{RN}$ reencodes the decoded bits, similarly to the classic STSK structure of [2]. Explicitly, according to the relationship of $b \cdot \log _{2} \mathcal{L}^{\prime}=\log _{2}(\mathcal{L} \cdot Q)$, the $\log _{2} \mathcal{L}$ bits of source information are mapped to an $\mathcal{L}$-PSK or $\mathcal{L}$-QAM symbol $s(k)$, whereas the remaining $\log _{2} Q$ bits select the $m$ th row vector $\boldsymbol{a}_{q}^{m}(k)$ of the $q$ th matrix from the set of $Q$ preassigned DMs $\boldsymbol{A}_{q} \in \mathbb{C}^{M \times T}$, $(q=1,2 \ldots, Q)$. The DMs are generated under the power constraint, as detailed in [2] and [27], i.e., $\operatorname{tr}\left(\boldsymbol{A}_{q}^{\mathrm{H}} \boldsymbol{A}_{q}\right)=T,(q=$ $1,2 \ldots, Q)$, where $T$ represents the number of time slots used in the specific STSK structure considered and $\operatorname{tr}(\bullet)$ and $\bullet H$ denote the trace and the Hermitian transpose of the matrix "•," respectively. Specifically, the $m$ th $\mathrm{RN}$ maps the decoded bits to a symbol vector $\boldsymbol{S}_{v_{i}}^{m}(k) \in \mathbb{C}^{1 \times T}, i=1,2$, which is given by $\boldsymbol{S}_{v_{i}}^{m}(k)=s(k) \boldsymbol{a}_{q}^{m}(k)$. Additionally, the activation/deactivation of the $m$ th $\mathrm{RN}$ may be represented by the parameter $\alpha_{m} \in\{0,1\}$, where we have $\alpha_{m}=0$ if a decoding error is identified by the CRC, hence resulting in the termination of relaying, and we have $\alpha_{m}=1$ if otherwise. Furthermore, the resultant cooperative scheme will be unambiguously referred to as a coherent cooperative MC STSK $(M, T, Q)$ scheme in conjunction with the associated $\mathcal{L}$-PSK or $\mathcal{L}$-QAM modulation.

\section{Receiver Model at Destination}

With the aid of the double-frame matched filter ${ }^{1}$ of [17] for a particular user $u$, and considering the FD representations of the signals and the FD channel response rather than the CIR, the signal received at the DN from the VAA-DN link during the frame-A and frame-B transmissions are given by [14], [17]

$$
\begin{aligned}
\boldsymbol{Y}_{v_{2} d}^{A}\left(k^{\prime}\right)= & \sum_{m=1}^{M}\left[\sqrt{G_{v_{2} d}} \alpha_{m} \tilde{h}_{v_{2} d}^{m}\left(k^{\prime}\right)\left[C_{B}\left(s_{f}\right) \boldsymbol{S}_{v_{2}}^{m}\left(k-L_{f}\right)\right]\right] \\
& +\tilde{\boldsymbol{N}}_{v_{2} d}^{\prime}\left(k^{\prime}\right) \\
= & \sqrt{G_{v_{2} d}} \tilde{\boldsymbol{H}}_{v_{2} d}^{\prime}\left(k^{\prime}\right)\left[C_{B}\left(s_{f}\right) \boldsymbol{A}_{q}\left(k-L_{f}\right) s\left(k-L_{f}\right)\right] \\
& +\tilde{\boldsymbol{N}}_{v_{2} d}^{\prime}\left(k^{\prime}\right) \\
\boldsymbol{Y}_{v_{1} d}^{B}\left(k^{\prime}\right)= & \sum_{m=1}^{M}\left[\sqrt{G_{v_{1} d}} \alpha_{m} \tilde{h}_{v_{1} d}^{m}\left(k^{\prime}\right)\left[C_{A}\left(s_{f}\right) \boldsymbol{S}_{v_{1}}^{m}\left(k-L_{f}\right)\right]\right] \\
& +\tilde{\boldsymbol{N}}_{v_{1} d}^{\prime}\left(k^{\prime}\right) \\
= & \sqrt{G_{v_{1} d}} \tilde{\boldsymbol{H}}_{v_{1} d}^{\prime}\left(k^{\prime}\right)\left[C_{A}\left(s_{f}\right) \boldsymbol{A}_{q}\left(k-L_{f}\right) s\left(k-L_{f}\right)\right] \\
& +\tilde{\boldsymbol{N}}_{v_{1} d}^{\prime}\left(k^{\prime}\right)
\end{aligned}
$$

${ }^{1} \mathrm{~A}$ filter matched to $C_{A}^{u}$ is employed during frame-A, whereas a filter matched to $C_{B}^{u}$ is employed during the next consecutive frame-B transmission. Application of this strategy helps to detect signals during a particular frame, considerably suppressing the SR-induced interference. where we have

$$
\begin{gathered}
\tilde{\boldsymbol{H}}_{v_{i} d}^{\prime}\left(k^{\prime}\right) \triangleq\left[\alpha_{1} \tilde{h}_{v_{i} d}^{1}\left(k^{\prime}\right), \ldots, \alpha_{M} \tilde{h}_{v_{i} d}^{M}\left(k^{\prime}\right)\right] \in \mathbb{C}^{1 \times M} \\
\boldsymbol{A}_{q}\left(k-L_{f}\right)=\left[\begin{array}{c}
\boldsymbol{a}_{q}^{1}\left(k-L_{f}\right) \\
\vdots \\
\boldsymbol{a}_{q}^{M}\left(k-L_{f}\right)
\end{array}\right] \in \mathbb{C}^{M \times T} .
\end{gathered}
$$

The FD channel coefficients $\tilde{h}_{v_{i} d}^{m}\left(k^{\prime}\right)$ and the noise components $\tilde{\mathbf{N}}_{v_{i} d}^{\prime}\left(k^{\prime}\right)$ for $i=1,2$ and $m=1,2, \ldots, M$ obey the complexvalued Gaussian distributions of $\mathcal{C N}\left(0, \sigma_{v_{i} d}^{2}\right)$ and $\mathcal{C N}\left(0, N_{0}\right)$, respectively.

Applying the vectorial stacking operation $\operatorname{vec}(\cdot)$ to both sides of (5) and (6), we arrive at the linearized VAA-DN link output signals, which is similar to the LDCs of [6]

$$
\begin{aligned}
\overline{\boldsymbol{Y}}_{v_{2} d}^{A}\left(k^{\prime}\right)= & \sqrt{G_{v_{2} d}} \overline{\boldsymbol{H}}_{v_{2} d}^{\prime}\left(k^{\prime}\right) C_{B}\left(s_{f}\right) \chi \boldsymbol{K}\left(k-L_{f}\right) \\
& +\overline{\boldsymbol{N}}_{v_{2} d}^{\prime}\left(k^{\prime}\right) \\
\overline{\boldsymbol{Y}}_{v_{1} d}^{B}\left(k^{\prime}\right)= & \sqrt{G_{v_{1} d}} \overline{\boldsymbol{H}}_{v_{1} d}^{\prime}\left(k^{\prime}\right) C_{A}\left(s_{f}\right) \chi \boldsymbol{K}\left(k-L_{f}\right) \\
& +\overline{\boldsymbol{N}}_{v_{1} d}^{\prime}\left(k^{\prime}\right)
\end{aligned}
$$

where we have

$$
\begin{aligned}
\overline{\boldsymbol{Y}}_{v_{2} d}^{A}\left(k^{\prime}\right) & =\operatorname{vec}\left(\boldsymbol{Y}_{v_{2} d}^{A}\left(k^{\prime}\right)\right) \in \mathbb{C}^{T \times 1} \\
\overline{\boldsymbol{Y}}_{v_{1} d}^{B}\left(k^{\prime}\right) & =\operatorname{vec}\left(\boldsymbol{Y}_{v_{1} d}^{B}\left(k^{\prime}\right)\right) \in \mathbb{C}^{T \times 1} \\
\chi & \triangleq\left[\operatorname{vec}\left(\boldsymbol{A}_{1}\right), \ldots, \operatorname{vec}\left(\boldsymbol{A}_{Q}\right)\right] \in \mathbb{C}^{M T \times Q} \\
\overline{\boldsymbol{H}}_{v_{i} d}^{\prime}\left(k^{\prime}\right) & \triangleq \sqrt{G_{v_{i} d}}\left[\boldsymbol{I}_{T} \otimes \tilde{\boldsymbol{H}}_{v_{i} d}^{\prime}\left(k^{\prime}\right)\right] \in \mathbb{C}^{T \times M T} \\
\boldsymbol{K}\left(k-L_{f}\right) & \triangleq[\underbrace{0, \ldots, 0}_{q-1}, s\left(k-L_{f}\right), \underbrace{0, \ldots, 0}]^{T} \in \mathbb{C}^{Q \times 1} \\
\overline{\boldsymbol{N}}_{v_{i} d}^{\prime}\left(k^{\prime}\right) & =\operatorname{vec}\left(\tilde{\boldsymbol{N}}_{v_{i} d}^{\prime}\left(k^{\prime}\right)\right) \in \mathbb{C}^{T \times 1}, \quad i=1,2 .
\end{aligned}
$$

Here, the equivalent signal vector $\boldsymbol{K}\left(k-L_{f}\right)$ has only a single nonzero symbol component $s\left(k-L_{f}\right)$ placed in the $q$ th position, $\boldsymbol{I}_{T} \in \mathbb{C}^{T \times T}$ is the identity matrix, $\otimes$ represents the Kronecker product, and $\bullet^{T}$ denotes the transpose of the matrix “•."

The combined received signal at the DN during both frame-A and frame-B transmissions is constituted by the superposition of the signals arriving from the SN-DN link and VAA-DN links, which can be expressed as [17]

$$
\begin{aligned}
\boldsymbol{Y}^{A}\left(k^{\prime}\right)= & \tilde{h}_{s d}\left(k^{\prime}\right) C_{A}\left(s_{f}\right) \boldsymbol{S}_{s}(k)+\tilde{\boldsymbol{N}}_{d}\left(k^{\prime}\right) \\
& +\sqrt{G_{v_{2} d}} \tilde{\boldsymbol{H}}_{v_{2} d}^{\prime}\left(k^{\prime}\right) C_{B}\left(s_{f}\right) \boldsymbol{A}_{q}\left(k-L_{f}\right) s\left(k-L_{f}\right) \\
& +\tilde{\boldsymbol{N}}_{v_{2} d}^{\prime}\left(k^{\prime}\right) \\
\boldsymbol{Y}^{B}\left(k^{\prime}\right)= & \tilde{h}_{s d}\left(k^{\prime}\right) C_{B}\left(s_{f}\right) \boldsymbol{S}_{s}(k)+\tilde{\boldsymbol{N}}_{d}\left(k^{\prime}\right) \\
& +\sqrt{G_{v_{1}}} \tilde{\boldsymbol{H}}_{v_{1} d}^{\prime}\left(k^{\prime}\right) C_{A}\left(s_{f}\right) \boldsymbol{A}_{q}\left(k-L_{f}\right) s\left(k-L_{f}\right) \\
& +\tilde{\boldsymbol{N}}_{v_{1} d}^{\prime}\left(k^{\prime}\right) .
\end{aligned}
$$




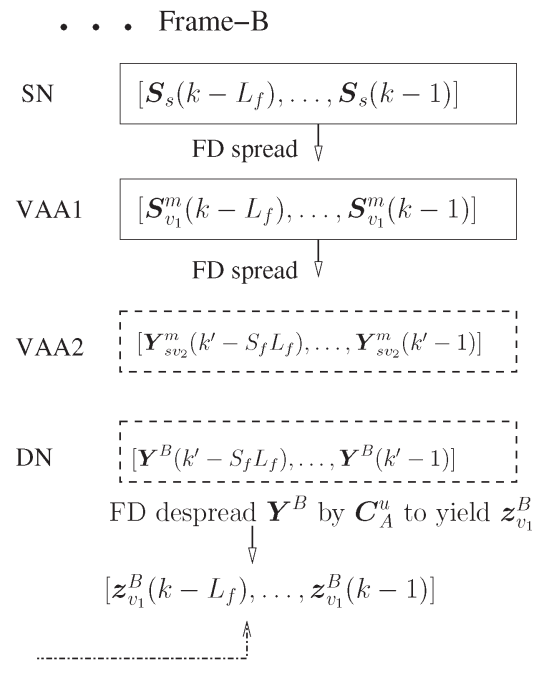

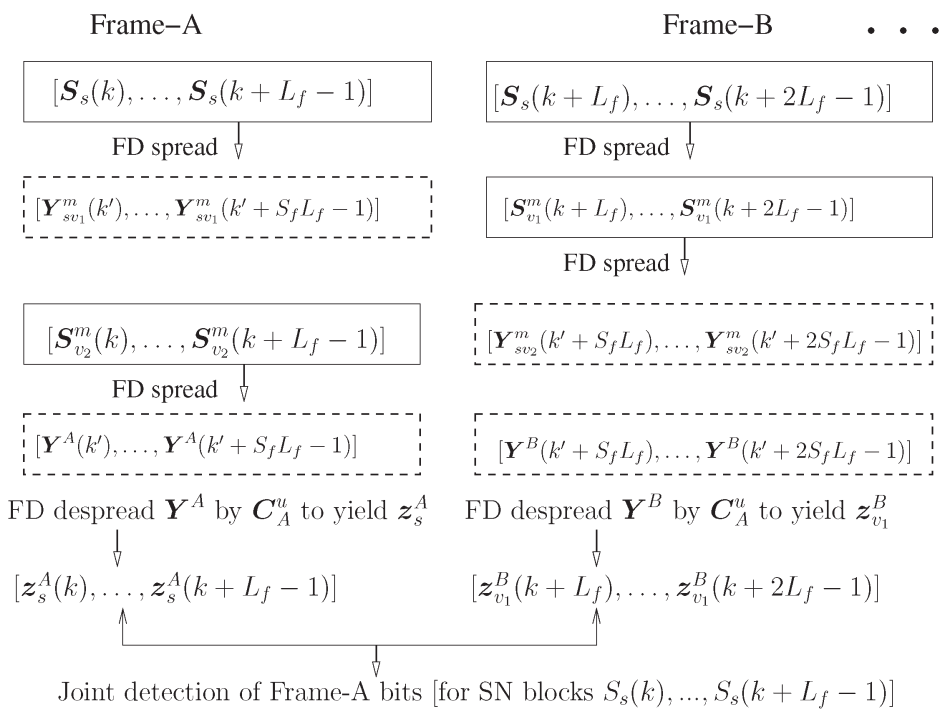

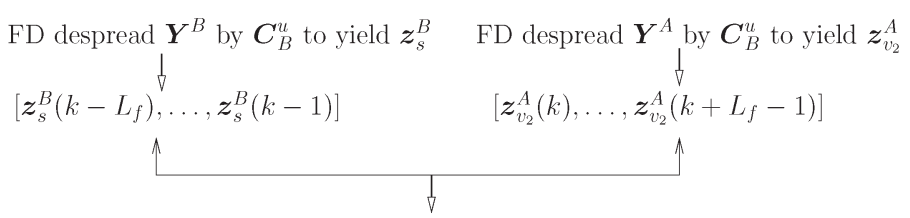

Joint detection of Frame-B bits [for SN blocks $\left.S_{s}\left(k-L_{f}\right), \ldots, S_{s}(k-1)\right]$

Fig. 3. Proposed SR-based cooperative STSK protocol to conceive the joint ML detector using the different transmitted and received symbol blocks of the corresponding frames. The solid box represents that the related node is transmitting, whereas signal reception at a particular node is indicated by the dashed box.

Now, employing the double-frame matched-filter-based despreading and defining the equivalent SN-DN channel transfer function by

$\bar{h}_{s d}(k) \triangleq \frac{1}{S_{f}}\left[\tilde{h}_{s d}\left(k^{\prime}\right)+\tilde{h}_{s d}\left(k^{\prime}+1\right)+\cdots+\tilde{h}_{s d}\left(k^{\prime}+S_{f}-1\right)\right]$

and the equivalent VAA2-DN channel matrix by

$$
\begin{array}{r}
\overline{\boldsymbol{H}}_{v_{2} d}(k) \triangleq \frac{1}{S_{f}}\left[\overline{\boldsymbol{H}}_{v_{2} d}^{\prime}\left(k^{\prime}\right)+\overline{\boldsymbol{H}}_{v_{2} d}^{\prime}\left(k^{\prime}+1\right)+\cdots\right. \\
\left.\cdots+\overline{\boldsymbol{H}}_{v_{2} d}^{\prime}\left(k^{\prime}+S_{f}-1\right)\right]
\end{array}
$$

where $k$ is related to $k^{\prime}$ by $k=\left\lceil k^{\prime} / S_{f}\right\rceil$, and $\lceil\cdot\rceil$ denotes the ceiling $(\cdot)$ operator. The pair of despread signals that gleaned from the SN-DN and the VAA2-DN links can be extracted from $\boldsymbol{Y}^{A}\left(k^{\prime}\right)$ during the transmission of frame-A, which is given by

$$
\begin{aligned}
& \boldsymbol{z}_{s}^{A}(k)=\bar{h}_{s d}(k) \boldsymbol{S}_{s}(k)+\boldsymbol{I}_{v_{2}}(k)+\boldsymbol{N}_{d}(k) \\
& \overline{\boldsymbol{z}}_{v_{2}}^{A}(k)=\overline{\boldsymbol{H}}_{v_{2} d}(k) \boldsymbol{\chi} \boldsymbol{K}\left(k-L_{f}\right)+\boldsymbol{I}_{s}(k)+\boldsymbol{N}_{v_{2} d}(k) .
\end{aligned}
$$

Similarly, the despread signals from $\boldsymbol{Y}^{B}\left(k^{\prime}\right)$ during frame-B's transmission may be expressed as

$$
\begin{aligned}
& \boldsymbol{z}_{s}^{B}(k)=\bar{h}_{s d}(k) \boldsymbol{S}_{s}(k)+\boldsymbol{I}_{v_{1}}(k)+\boldsymbol{N}_{d}(k) \\
& \overline{\boldsymbol{z}}_{v_{1}}^{B}(k)=\overline{\boldsymbol{H}}_{v_{1} d}(k) \boldsymbol{\chi} \boldsymbol{K}\left(k-L_{f}\right)+\boldsymbol{I}_{s}(k)+\boldsymbol{N}_{v_{1} d}(k)
\end{aligned}
$$

where $\overline{\boldsymbol{z}}_{v_{2}}^{A}(k)$ and $\overline{\boldsymbol{z}}_{v_{2}}^{A}(k)$ are the vectorially stacked despread signal from the VAA-DN links; $\boldsymbol{I}_{s}(k), \boldsymbol{I}_{v_{1}}(k)$, and $\boldsymbol{I}_{v_{2}}(k)$ are the interference terms that are substantially mitigated by the specific spread-despread regime, particularly at a high $S_{f}$ and
$\boldsymbol{N}_{d}(k) ; \boldsymbol{N}_{v_{1} d}(k)$ and $\boldsymbol{N}_{v_{2} d}(k)$ are the additive white Gaussian noise (AWGN) terms imposed on the corresponding signals.

\section{JOINT Single-STREAM MAXIMUM-LiKELIHOOD Detection of the Proposed Cooperative Scheme}

The joint single-stream ML detector of our scheme detects the source information from the signals received from both the SN-DN and VAA-DN links, as detailed in [13] and [14], but takes the delay of the relayed frame due to both SR and the double-frame FD despreading [17] into account.

The different stages of the joint detection procedure appropriately combining the components of the transmitted, received, and despread signals during the different transmission frames are visualized in Fig. 3. It is plausible that due to the inherent nature of SR, the two replicas of the same frame, which are broadcast through the direct SN-DN link during the broadcast phase, with its counterpart forwarded by the VAA through the VAA-DN link in the consecutive cooperative phase, cannot arrive at the DN at the same time. Hence, as shown in Fig. 3, the joint detection of the transmitted information has to be carried out over two consecutive frames of the FD despread received signals.

Thus, the joint detection of the source information on a user that is broadcasted by the SN during Frame-A is performed by combining the two replicas mentioned previously. This combination yields the Frame-A received sequence $Z^{A}(k)$, which may be formally expressed as [13], [14]

$$
\begin{aligned}
\boldsymbol{Z}^{A}(k) & \triangleq\left[\begin{array}{c}
\boldsymbol{z}_{s}^{A}(k) \\
\overline{\boldsymbol{z}}_{v_{1}}^{B}\left(k+L_{f}\right)
\end{array}\right] \\
& =\boldsymbol{H}_{J}^{A}(k) \overline{\boldsymbol{S}}_{s}(k)+\boldsymbol{N}_{J}(k) \in \mathbb{C}^{(b+T) \times 1}
\end{aligned}
$$


where we have

$$
\begin{aligned}
\overline{\boldsymbol{S}}_{s}(k) & \triangleq\left[\begin{array}{l}
\boldsymbol{S}_{s}(k) \\
\boldsymbol{K}(k)
\end{array}\right] \in \mathbb{C}^{(b+Q) \times 1} \\
\boldsymbol{N}_{J}(k) & =\left[\begin{array}{c}
\boldsymbol{I}_{v_{2}}(k)+\boldsymbol{N}_{d}(k) \\
\boldsymbol{I}_{s}\left(k+L_{f}\right)+\boldsymbol{N}_{v_{1} d}\left(k+L_{f}\right)
\end{array}\right] \in \mathbb{C}^{(b+T) \times 1}
\end{aligned}
$$

and the combined FD channel transfer matrix, i.e.,

$$
\boldsymbol{H}_{J}(k) \triangleq\left[\begin{array}{c:c}
\bar{h}_{s d}(k) \boldsymbol{I}_{b} & \mathbf{0}_{b \times Q} \\
\hdashline \mathbf{0}_{T \times b} & \overline{\boldsymbol{H}}_{v_{1} d}\left(k+L_{f}\right) \boldsymbol{\chi}
\end{array}\right] \in \mathbb{C}^{(b+T) \times(b+Q)}
$$

has two submatrices expressed by $\bar{h}_{s d}(k) \boldsymbol{I}_{b} \in \mathbb{C}^{b \times b}$ and $\overline{\boldsymbol{H}}_{v_{1} d}\left(k+L_{f}\right) \chi \in \mathbb{C}^{T \times Q}$, respectively, and two zero matrices.

Additionally, the equivalent transmit signal vector of the $k$ th block $\boldsymbol{K}(k)$ in (26) using the $q$ th DM and the $l$ th constellation symbol $s_{l}$ may be expressed by $\boldsymbol{K}_{l, q}=$ $[\underbrace{0, \ldots, 0}_{q-1}, s_{l}, \underbrace{0, \ldots, 0}_{Q-q}]^{T} \in \mathbb{C}^{Q \times 1}$.

If the SR-imposed interference components $\boldsymbol{I}_{v_{2}}$ and $\boldsymbol{I}_{s}$ are approximated by noise processes, the equivalent noise process $N_{J}$ can be assumed to be Gaussian distributed having the same variance as $\boldsymbol{I}_{v_{2}}$ and $\boldsymbol{I}_{s}$.

The joint ML detector conceived for our cooperative scheme estimates the source information during Frame-A transmission of a particular user based on the FD despread direct SN-DN frame and on the FD despread frame arriving via the VAA1-DN link, which may be formulated as [14], [22]

$$
\begin{aligned}
& {[\hat{q}(k), \hat{l}(k)] } \\
&=\underset{q, l}{\arg \min }\{\left\{\left\|\boldsymbol{Z}^{A}(k)-\boldsymbol{H}_{J}^{A}(k) \overline{\boldsymbol{S}}_{s}^{q, l}\right\|^{2}\right\} \\
&=\underset{q, l}{\arg \min }\left\{\left\|\boldsymbol{z}_{s}^{A}(k)-\bar{h}_{s d}(k) \boldsymbol{S}_{s}^{q, l}\right\|^{2}\right. \\
&\left.+\left\|\overline{\boldsymbol{z}}_{v_{1}}^{B}\left(k+L_{f}\right)-s_{l}\left(\overline{\boldsymbol{H}}_{v_{1} d}\left(k+L_{f}\right) \boldsymbol{\chi}\right)_{q}\right\|^{2}\right\}
\end{aligned}
$$

where $\|\bullet\|$ represents the Euclidean norm of the matrix "•," $\boldsymbol{S}_{s}^{q, l}$ and $\overline{\boldsymbol{S}}_{s}^{q, l}$ are the legitimate values of the symbol blocks $\boldsymbol{S}_{s}(k)$ and $\overline{\boldsymbol{S}}_{s}(k)$ specified by the indices $(q, l)$, and $\left(\overline{\boldsymbol{H}}_{v_{1} d}(k+\right.$ $\left.\left.L_{f}\right) \boldsymbol{\chi}\right)_{q}$ indicates the $q$ th column of $\overline{\boldsymbol{H}}_{v_{1} d}\left(k+L_{f}\right) \boldsymbol{\chi}$. As shown in Fig. 3, the joint ML detector for the next consecutive frame can be formulated from $\boldsymbol{z}_{s}^{B}\left(k+L_{f}\right)$ and $\boldsymbol{z}_{v_{2}}^{A}\left(k+2 L_{f}\right)$. Since the signal vectors received from the RNs during the VAA's cooperation phase are composed of the row vectors from a single DM, the joint detection scheme remains immune to the interstream interference.

In a multiuser scenario, the received sequence will be the superposition of the sequences corresponding to the individual users. Since the orthogonality of the spreading sequences of different users is destroyed by the dispersive channels, multiuser interference (MUI) is imposed. Upon reinstating the user index $u$, we can formulate the superposed destination signal with the aid of (3) and (10) in a form similar to (25), which has the additional MUI term as follows:

$$
\begin{aligned}
& \boldsymbol{Y}^{A}\left(k^{\prime}\right) \\
& =\sum_{u=1}^{U}\left[\begin{array}{c}
\boldsymbol{Y}_{s d}^{A, u}\left(k^{\prime}\right) \\
\overline{\boldsymbol{Y}}_{v_{1 d}}^{,, u}\left(k^{\prime}+L_{f} \cdot S_{f}\right)
\end{array}\right] \\
& =\underbrace{\boldsymbol{H}_{J^{\prime}}^{A, v}\left(k^{\prime}\right) \overline{\boldsymbol{S}}_{c}^{v}\left(k^{\prime}\right)}_{\text {desired user's signal }}+\underbrace{\sum_{\substack{u=1 \\
u \neq v}}^{U} \boldsymbol{H}_{J^{\prime}}^{A, u}\left(k^{\prime}\right) \overline{\boldsymbol{S}}_{c}^{u}\left(k^{\prime}\right)}_{\text {MUI }}+\underbrace{\boldsymbol{N}_{J}^{u}\left(k^{\prime}\right)}_{\text {additive noise }}
\end{aligned}
$$

where $\overline{\boldsymbol{S}}_{c}^{u}\left(k^{\prime}\right), \boldsymbol{N}_{J}^{u}\left(k^{\prime}\right)$, and $\boldsymbol{H}_{J^{\prime}}^{A, u}\left(k^{\prime}\right)$ are defined similar to $\overline{\boldsymbol{S}}_{s}(k), \boldsymbol{N}_{J}(k)$, and $\boldsymbol{H}_{J}^{A}(k)$ in (26), (27) and (28), respectively, but refer to the transmission of the spread symbol block indexed by $k^{\prime}$ of user $u$. Furthermore, the desired user has been denoted by $v$, the generalized user by $u$, and $u \neq v$ represents the interfering user.

A MUD [26], [28] combined with the single-stream ML detector in [1] and [2] may be used in the multiuser scenario for jointly detecting the information on the different users. Since the source information on the users in the $k$ th symbol block $\boldsymbol{S}_{s}^{u}(k)$ is spread over $S_{f}$ blocks from $\boldsymbol{Y}^{A}\left(k S_{f}+1\right)$ to $\boldsymbol{Y}^{A}\left([k+1] S_{f}\right)$, the ML-MUD may be formulated as in (31), shown below, to jointly estimate the set of indices for the DM, i.e., $\boldsymbol{q}(k)=\left\{q^{0}(k), \ldots, q^{(U-1)}(k)\right\}$, and the constellation symbol, i.e., $l_{c}(k)=\left\{l_{c}^{0}(k), \ldots, l_{c}^{(U-1)}(k)\right\}$. In (31), the transmitted indices for the $u$ th user are represented by $q^{u}$ and $l_{c}^{u}$, respectively; $s_{l_{u}^{u}}^{u}$ denotes the $l_{c}^{u}$ th constellation symbol of user $u$; and $(\bullet)_{q^{u}}$ indicates the $q^{u}$ th column of the matrix " $\bullet$."

Equations (30) and (31) explicitly portray the MUI, but the MUD complexity escalates upon increasing the number of users, despite the fact that each user activates a single DM at a time, as indicated by the $q^{u}$ th column of the dispersion characterizing matrix $\chi$ in

$$
\begin{aligned}
& \left(\hat{\boldsymbol{q}}(k), \hat{\boldsymbol{l}}_{c}(k)\right) \\
& =\arg \min _{\boldsymbol{q}, l_{c}} \sum_{s_{f}=1}^{S_{f}}\left\{\| \boldsymbol{Y}_{s d}^{A, u}\left(k \cdot S_{f}+s_{f}\right)\right. \\
& -\sum_{u=1}^{U} \tilde{h}_{s d}^{u}\left(k \cdot S_{f}+s_{f}\right) C_{A}^{u}\left(s_{f}\right) \boldsymbol{S}_{s}^{q^{u}, l_{c}^{u}} \|^{2} \\
& +\| \overline{\boldsymbol{Y}}_{v_{1 d}}^{B, u}\left(\left[k+L_{f}\right] \cdot S_{f}+s_{f}\right) \\
& \quad-\sum_{u=1}^{U} C_{B}^{u}\left(s_{f}\right) s_{l_{c}^{u}}^{u} \\
& \left.\quad \times\left(\overline{\boldsymbol{H}}_{v_{1} d}^{u}\left(\left[k+L_{f}\right] \cdot S_{f}+s_{f}\right) \boldsymbol{\chi}\right)_{q^{u}} \|^{2}\right\}
\end{aligned}
$$




\section{Design of a COOPERATIVE NonCOHERENT MulticARrier SPACE-TIME SHIFT KEYING SCHEME}

Here, we introduce our differentially encoded and noncoherently detected MC cooperative STSK scheme relying on SR dispensing with any channel estimation (CE). This arrangement retains all the benefits of its coherent counterpart but typically requires a 3-dB higher power.

Regarding our differential encoding scheme, the following points are worth mentioning with special emphasis.

1) The DMs we use for the differential cooperative STSK scheme are directly generated unitary matrices $\boldsymbol{A}_{q}(q=$ $1, \ldots, Q)$, which allow us to avoid the nonlinear Cayley transform of [2].

2) The differential encoding requires satisfying the STSKrelated condition of relying on $M=T$, so that the resultant STSK signaling blocks are $(T \times T)$-element square matrices.

3) Differential encoding of the MC-based system can be performed either in the TD (differential encoding across the consecutive symbols of the same subcarrier) or in the FD (differential encoding across the symbols of the adjacent subcarriers of the same MC-CDMA block). We opted for invoking the TD approach because our scheme was conceived for frequency-selective channels, which exhibit flat fading for the individual subcarriers, whereas the FD channel envelope of the adjacent subcarriers might be different.

In the differential scheme, we utilize $\mathcal{L}^{\prime}$-differential PSK modulation at the $\mathrm{SN}$. The spread blocks $\boldsymbol{S}_{c}\left(k^{\prime}\right)=\left[s_{c, 1}\left(k^{\prime}\right), \ldots\right.$, $\left.s_{c, b}\left(k^{\prime}\right)\right]^{T}$ are obtained from $\boldsymbol{S}_{s}(k)$ by $\boldsymbol{S}_{c}\left(k^{\prime}\right)=\left[C_{A}\left(s_{f}\right) \boldsymbol{S}_{s}(k)\right]$ or by $\boldsymbol{S}_{c}\left(k^{\prime}\right)=\left[C_{B}\left(s_{f}\right) \boldsymbol{S}_{s}(k)\right]$, where $s_{f}=1,2, \ldots, S_{f}$ and $k^{\prime}=k \times S_{f}+s_{f}$, depending on which frame is being transmitted. The consecutive spread blocks under the same subcarrier are placed $N_{c}$ blocks apart in any transmission frame, where $N_{c}$ is the number of subcarriers. Hence, the differentially encoded transmit block $S_{s}^{\prime}\left(k^{\prime}\right) \in \mathbb{C}^{b \times 1}$ for $k^{\prime}=-\left(N_{c}-1\right)$, $\ldots, 0,1,2, \ldots, S_{f} L_{f}$ at the $\mathrm{SN}$ of each transmission frame is related to $\boldsymbol{S}_{c}\left(k^{\prime}\right)$ by

$\boldsymbol{S}_{s}^{\prime}\left(k^{\prime}\right)= \begin{cases}{\left[s_{1}^{\prime}\left(k^{\prime}\right), \ldots, s_{b}^{\prime}\left(k^{\prime}\right)\right]^{T},} & k^{\prime}=1,2, \ldots, S_{f} L_{f} \\ {[\underbrace{1,1, \ldots, 1}_{b}]^{T},} & k^{\prime}=-\left(N_{c}-1\right), \ldots, 1,0\end{cases}$

where $s_{j}^{\prime}\left(k^{\prime}\right)=s_{j}^{\prime}\left(k^{\prime}-N_{c}\right) s_{c, j}\left(k^{\prime}\right), j=1,2, \ldots, b$. Taking differential decoding into consideration, the FD received signals $\boldsymbol{Y}_{s d}^{A}\left(k^{\prime}\right) \in \mathbb{C}^{b \times 1}$ and $\boldsymbol{Y}_{s d}^{B}\left(k^{\prime}\right) \in \mathbb{C}^{b \times 1}$ at the DN from the direct SN-DN link during Frame-A and Frame-B transmissions are then

$$
\begin{aligned}
\boldsymbol{Y}_{A}^{\prime}\left(k^{\prime}\right) & =\boldsymbol{H}_{s d}^{A}\left(k^{\prime}\right)\left[C_{A}\left(s_{f}\right) \boldsymbol{S}_{s}(k)\right]+\tilde{\boldsymbol{N}}_{d}\left(k^{\prime}\right) \\
\boldsymbol{Y}_{B}^{\prime}\left(k^{\prime}\right) & =\boldsymbol{H}_{s d}^{B}\left(k^{\prime}\right)\left[C_{B}\left(s_{f}\right) \boldsymbol{S}_{s}(k)\right]+\tilde{\boldsymbol{N}}_{d}\left(k^{\prime}\right)
\end{aligned}
$$

respectively, where we make the substitutions $\boldsymbol{H}_{s d}^{A}\left(k^{\prime}\right)=$ $\operatorname{diag}\left\{Y_{A}^{\prime}\left(k^{\prime}-N_{c}\right)[1], \ldots, Y_{A}^{\prime}\left(k^{\prime}-N_{c}\right)[b]\right\} \in \mathbb{C}^{b \times b}$ and $\boldsymbol{H}_{s d}^{B}\left(k^{\prime}\right)=$ $\operatorname{diag}\left\{Y_{B}^{\prime}\left(k^{\prime}-N_{c}\right)[1], \ldots, Y_{B}^{\prime}\left(k^{\prime}-N_{c}\right)[b]\right\} \in \mathbb{C}^{b \times b}, Y_{A}^{\prime}\left(k^{\prime}-\right.$ $\left.N_{c}\right)[1], \ldots, Y_{A}^{\prime}\left(k^{\prime}-N_{c}\right)[b]$ are the $b$ symbols of the re- ceived block $\boldsymbol{Y}_{A}^{\prime}\left(k^{\prime}-N_{c}\right)$, and notation $\operatorname{diag}\{a[1], \ldots, a[b]\}$ represents a $(b \times b)$ diagonal matrix with diagonal entries $a[1], \ldots, a[b]$.

The RN $m$ of VAA $i$ transmits only the $m$ th row of the differentially encoded STSK codeword, whereas the STSK signaling block $\boldsymbol{X}(k)=s(k) \boldsymbol{A}_{q}(k) \in \mathbb{C}^{T \times T}$ is created from the correctly decoded bits at the $\mathrm{RN}$ by activating a single DM, i.e., $\boldsymbol{A}_{q}(k)(q=1, \ldots, Q)$ for the transmission of the $\mathcal{L}$-PSK or $\mathcal{L}$-QAM symbol, i.e., $s(k)=s_{l}$. The STSK space-time codeword $\boldsymbol{X}(k)$ is further FD spread to $\tilde{\boldsymbol{X}}\left(k^{\prime}\right)=C_{A}\left(s_{f}\right) \boldsymbol{X}(k) \in \mathbb{C}^{T \times T} \quad s_{f}=1,2, \ldots, S_{f}$ or to $\tilde{\boldsymbol{X}}\left(k^{\prime}\right)=C_{B}\left(s_{f}\right) \boldsymbol{X}(k) \in \mathbb{C}^{T \times T} s_{f}=1,2, \ldots, S_{f}$, depending on which frame is being transmitted, where $k=\left\lceil k^{\prime} / S_{f}\right\rceil$ and $s_{f}=\left(k^{\prime} \bmod S_{f}\right)$ ("mod" denotes the modulo operator). Therefore, for the VAA-DN link, we have the differentially encoded codeword $\boldsymbol{S}_{v_{i}}\left(k^{\prime}\right) \in \mathbb{C}^{T \times T}$ expressed by

$$
\boldsymbol{S}_{v_{i}}\left(k^{\prime}\right)= \begin{cases}\boldsymbol{S}_{v_{i}}\left(k^{\prime}-N_{c}\right) \tilde{\boldsymbol{X}}\left(k^{\prime}\right), & k^{\prime}=1,2, \ldots, S_{f} L_{f} \\ \boldsymbol{I}_{T}, & k^{\prime}=-\left(N_{c}-1\right), \ldots, 1,0\end{cases}
$$

for each transmit frame, where $\boldsymbol{I}_{T} \in \mathbb{C}^{T \times T}$ denotes the identity matrix.

Defining the signals received via the VAA-DN links $\boldsymbol{Y}_{v_{2} d}^{A}\left(k^{\prime}\right) \in \mathbb{C}^{1 \times T}$ and $\boldsymbol{Y}_{v_{1} d}^{B}\left(k^{\prime}\right) \in \mathbb{C}^{1 \times T}$ in terms of the relay activation parameter $\alpha_{m}$ as in (5) and (6), we have

$$
\begin{aligned}
\boldsymbol{Y}_{v_{2} d}^{A}\left(k^{\prime}\right)= & \boldsymbol{Y}_{v_{2} d}^{A}\left(k^{\prime}-N_{c}\right) C_{B}\left(s_{f}\right) \boldsymbol{X}\left(k-L_{f}\right) \\
& +\overline{\boldsymbol{N}}_{v_{2} d}^{\prime}\left(k^{\prime}\right) \\
\boldsymbol{Y}_{v_{1} d}^{B}\left(k^{\prime}\right)= & \boldsymbol{Y}_{v_{1} d}^{B}\left(k^{\prime}-N_{c}\right) C_{A}\left(s_{f}\right) \boldsymbol{X}\left(k-L_{f}\right) \\
& +\overline{\boldsymbol{N}}_{v_{1} d}^{\prime}\left(k^{\prime}\right)
\end{aligned}
$$

where $\overline{\boldsymbol{N}}_{v_{2} d}^{\prime}\left(k^{\prime}\right) \in \mathbb{C}^{1 \times T}$ and $\overline{\boldsymbol{N}}_{v_{1} d}^{\prime}\left(k^{\prime}\right) \in \mathbb{C}^{1 \times T}$ are the corresponding AWGN vector.

Replacing $\boldsymbol{Y}_{v_{2} d}^{A}\left(k^{\prime}-N_{c}\right)$ and $\boldsymbol{Y}_{v_{1} d}^{B}\left(k^{\prime}-N_{c}\right)$ by $\mathbb{H}_{v_{2} d}\left(k^{\prime}\right)$ and $\mathbb{H}_{v_{1} d}\left(k^{\prime}\right)$, respectively, for the differential scheme, the equivalent received signals $\overline{\boldsymbol{Y}}_{v_{2} d}^{A}\left(k^{\prime}\right)=\operatorname{vec}\left[\boldsymbol{Y}_{v_{2} d}^{A}\left(k^{\prime}\right)\right] \in \mathbb{C}^{T \times 1}$ and $\overline{\boldsymbol{Y}}_{v_{1} d}^{B}\left(k^{\prime}\right)=\operatorname{vec}\left[\boldsymbol{Y}_{v_{1} d}^{B}\left(k^{\prime}\right)\right] \in \mathbb{C}^{T \times 1}$ may be expressed as

$$
\begin{aligned}
& \overline{\boldsymbol{Y}}_{v_{2} d}^{A}\left(k^{\prime}\right)=\boldsymbol{H}_{v_{2} d}\left(k^{\prime}\right) C_{B}\left(s_{f}\right) \chi \boldsymbol{K}\left(k-L_{f}\right)+\breve{\boldsymbol{N}}_{v_{2} d}^{\prime}\left(k^{\prime}\right) \\
& \overline{\boldsymbol{Y}}_{v_{1} d}^{B}\left(k^{\prime}\right)=\boldsymbol{H}_{v_{1} d}\left(k^{\prime}\right) C_{A}\left(s_{f}\right) \chi \boldsymbol{K}\left(k-L_{f}\right)+\breve{\boldsymbol{N}}_{v_{1} d}^{\prime}\left(k^{\prime}\right)
\end{aligned}
$$

where $\boldsymbol{H}_{v_{2} d}\left(k^{\prime}\right)=\sqrt{G_{v_{i} d}}\left[\boldsymbol{I}_{T} \otimes \mathbb{H}_{v_{2} d}\left(k^{\prime}\right)\right] \in \mathbb{C}^{T \times M T}, \boldsymbol{H}_{v_{1} d}\left(k^{\prime}\right)=$ $\left.\boldsymbol{I}_{T} \otimes \mathbb{H}_{v_{1} d}\left(k^{\prime}\right)\right] \in \mathbb{C}^{T \times M T}, \breve{\boldsymbol{N}}_{v_{2} d}^{\prime}\left(k^{\prime}\right)=\operatorname{vec}\left[\overline{\boldsymbol{N}}_{v_{2} d}^{\prime}\left(k^{\prime}\right)\right] \in \mathbb{C}^{T \times 1}$, $\breve{\mathbf{N}}_{v_{1} d}^{\prime}\left(k^{\prime}\right)=\operatorname{vec}\left[\overline{\boldsymbol{N}}_{v_{1} d}^{\prime}\left(k^{\prime}\right)\right] \in \mathbb{C}^{T \times 1}$, and $M=T$.

Applying FD double-frame matched-filter-based despreading at the DN, we obtain

$$
\begin{aligned}
\boldsymbol{z}_{s}^{A}(k)= & \overline{\boldsymbol{H}}_{s d}^{A}(k) \boldsymbol{S}_{s}(k)+\boldsymbol{I}_{v_{2}}(k)+\boldsymbol{N}_{d}(k) \\
\overline{\boldsymbol{z}}_{v_{2}}^{A}(k)= & \breve{\boldsymbol{H}}_{v_{2} d}(k) \boldsymbol{\chi}\left(k-L_{f}\right) \\
& +\boldsymbol{I}_{s}(k)+\boldsymbol{N}_{v_{2} d}(k) \\
\boldsymbol{z}_{s}^{B}\left(k-L_{f}\right)= & \overline{\boldsymbol{H}}_{s d}^{B}\left(k-L_{f}\right) \boldsymbol{S}_{s}\left(k-L_{f}\right) \\
& +\boldsymbol{I}_{v_{1}}\left(k-L_{f}\right)+\boldsymbol{N}_{d}\left(k-L_{f}\right) \\
\overline{\boldsymbol{z}}_{v_{1}}^{B}\left(k+L_{f}\right)= & \breve{\boldsymbol{H}}_{v_{1} d}\left(k+L_{f}\right) \boldsymbol{\chi} \boldsymbol{K}(k) \\
& +\boldsymbol{I}_{s}\left(k+L_{f}\right)+\boldsymbol{N}_{v_{1} d}\left(k+L_{f}\right)
\end{aligned}
$$



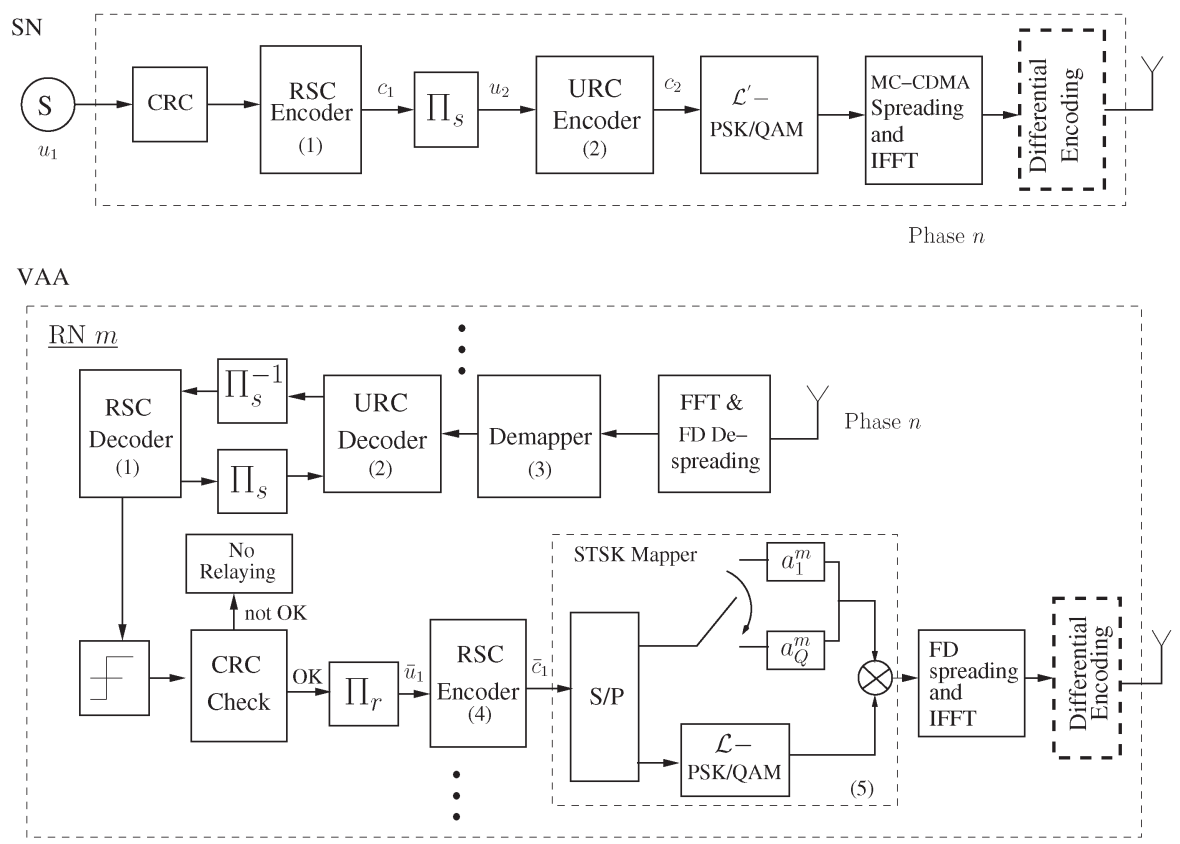

Phase $(n+1)$

Fig. 4. Transmission model of the near-capacity RSC and URC-aided SN and RNs of the cooperative MC scheme between two VAAs.

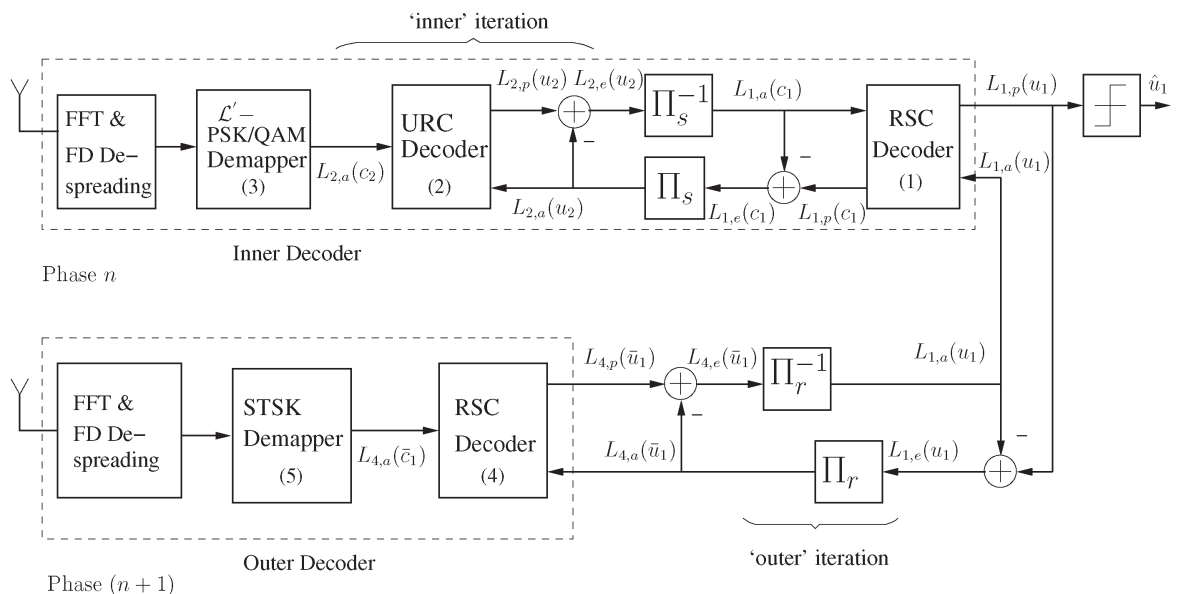

Fig. 5. Three-stage iterative detector at the destination.

where $\overline{\boldsymbol{H}}_{s d}^{A}(k), \overline{\boldsymbol{H}}_{s d}^{B}(k), \breve{\boldsymbol{H}}_{v_{1} d}(k)$, and $\breve{\boldsymbol{H}}_{v_{2} d}(k)$ are related to $\boldsymbol{H}_{s d}^{A}\left(k^{\prime}\right), \boldsymbol{H}_{s d}^{B}\left(k^{\prime}\right), \boldsymbol{H}_{v_{1} d}\left(k^{\prime}\right)$, and $\boldsymbol{H}_{v_{2} d}\left(k^{\prime}\right)$, respectively, in a similar manner, as in (19) and (20).

The joint ML detector of (29) can be now applied, employing $\boldsymbol{z}_{s}^{A}(k), \overline{\boldsymbol{z}}_{v_{1}}^{B}\left(k+L_{f}\right), \overline{\boldsymbol{H}}_{s d}^{A}(k)$, and $\breve{\boldsymbol{H}}_{v_{2} d}\left(k+L_{f}\right)$ to estimate the Frame-A information. For the estimation of Frame-B signal, the joint ML detector of (29) has to be applied employing $\boldsymbol{z}_{s}^{B}\left(k-L_{f}\right), \overline{\boldsymbol{z}}_{v_{2}}^{A}(k), \overline{\boldsymbol{H}}_{s d}^{B}\left(k-L_{f}\right)$, and $\breve{\boldsymbol{H}}_{v_{2} d}(k)$.

\section{Channel-Coded Soft-Decision Successive RELAYING-Aided Multicarrier CoOperative SPACE-TIME SHIFT KeYING}

Here, we propose the powerful channel-coded cooperative scheme shown in Figs. 4 and 5, which employs soft-decisionbased iterative detection. As demonstrated in Fig. 4, our transmitter consists of a three-stage serially concatenated recursive systematic convolutional (RSC) and unity-rate-coding-aided (URC) $\mathcal{L}$-PSK/QAM mapper followed by the MC-CDMA FD spreader plus the inverse fast Fourier transform (IFFT)module-based modulator. At each of the RNs of each VAA, the same two-component serially concatenated RSC-URC scheme is amalgamated with our MC-based STSK despread/spread and de/encode regime. The blocks $\Pi_{s}$ and $\Pi_{r}$ in Fig. 4 represent the random bit interleavers used both at the $\mathrm{SN}$ and at each $\mathrm{RN}$ of each VAA. Our soft-decision-based scheme can be employed for both the coherent and differential cooperative MC STSK arrangements, where the latter has a differential encoding block before the transmit antenna. The differential encoding block is shown as a dotted line in Fig. 4.

The iterative receiver of the destination in our three-stage cooperative arrangement is portrayed in Fig. 5. The signals received after FFT and FD despreading during phase $n$ at the DN are iteratively detected. Except for the first and last phases 
of the $(N+1)$-phase relaying protocol, the $\mathrm{DN}$ jointly detects the information on phase $n$ gleaned from the signals received from the $\mathrm{SN}$, in addition to that acquired via the VAA during phase $(n+1)$. As such, the relayed signal of frame $(n+1)$ is jointly detected with the SN's signal of frame $n$, whereas that from the relayed frame $n$ is treated as interference.

The conditional probability $p\left(\boldsymbol{Z}^{A}(k) \mid \overline{\boldsymbol{S}}_{s}^{q, l}, \boldsymbol{H}_{J}(k)\right)$ can be deduced according to the system model described by (25) as

$p\left(\boldsymbol{Z}^{A}(k) \mid \overline{\boldsymbol{S}}_{s}^{q, l}, \boldsymbol{H}_{J}(k)\right)=\frac{1}{\left(\pi N_{0}\right)^{b+T}} e^{-\frac{\left\|\boldsymbol{z}^{A}(k)-\boldsymbol{H}_{J}^{A}(k) \overline{\boldsymbol{S}}_{s}^{q, l}\right\|^{2}}{N_{0}}}$

where

$$
\begin{aligned}
& \left\|\boldsymbol{Z}^{A}(k)-\boldsymbol{H}_{J}^{A}(k) \overline{\boldsymbol{S}}_{s}^{q, l}\right\|^{2} \\
& \quad=\left\|\left[\begin{array}{c}
\boldsymbol{z}_{s}^{A}(k) \\
\overline{\boldsymbol{z}}_{v_{1}}^{B}\left(k+L_{f}\right)
\end{array}\right]-\left[\begin{array}{c}
\bar{h}_{s d}(k) \boldsymbol{S}_{s}^{q, l} \\
\overline{\boldsymbol{H}}_{v_{1} d}\left(k+L_{f}\right) \boldsymbol{\chi} \boldsymbol{K}_{l, q}
\end{array}\right]\right\|^{2}
\end{aligned}
$$

and $\boldsymbol{S}_{s}^{q, l}(k)$ and $\overline{\boldsymbol{S}}_{s}^{q, l}(k)$ represent the symbol blocks, as discussed in Section III and specified by indices $(q, l)$. For the differential scheme, the substitutions detailed in Section IV have to be made.

We note that, if at stage $n$, the equivalent FD received signal $\boldsymbol{z}_{s}^{A}(k)$ received directly from the SN carries $B$ channel-coded bits $b=\left[b_{1}, b_{2}, \ldots, b_{B}\right]$, then the extrinsic log-likelihood ratio (LLR) of bits $b_{k}, k=1, \ldots, B$ gleaned from the demapper can be expressed as [27], [29]

$$
L_{1, E}\left(b_{k}\right)=\ln \frac{\sum_{\boldsymbol{S}_{s}^{q, l} \in \boldsymbol{S}_{1}} e^{-\frac{\left\|z_{s}^{A}(k)-\bar{h}_{s d}(k) \boldsymbol{S}_{s}^{q, l}\right\|^{2}}{N_{0}}}+\sum_{j \neq k} b_{j} L_{1, A}\left(b_{j}\right)}{\sum_{\boldsymbol{S}_{s}^{q, l} \in \boldsymbol{S}_{0}} e^{-\frac{\left\|z_{s}^{A}(k)-\bar{h}_{s d}(k) \boldsymbol{S}_{s}^{q, l}\right\|^{2}}{N_{0}}}+\sum_{j \neq k} b_{j} L_{1, A}\left(b_{j}\right)}
$$

where $\boldsymbol{S}_{1}$ and $\boldsymbol{S}_{0}$ represent the subsets of the legitimate signal vectors transmitted directly by the SN-DN link $\boldsymbol{S}_{s}(k)$ corresponding to bits $b_{k}=1$ and $b_{k}=0$, respectively, and $L_{1, A}\left(b_{j}\right)$ is the a priori LLR corresponding to the "inner" decoder bits $b_{j}$.

Similarly, the $(n+1)$-stage LLRs acquired from the VAA demapper for the same bits $b_{k}, k=1, \ldots, B$ obtained from $\boldsymbol{z}_{v_{1}}^{B}\left(k+L_{f}\right)$ can be formulated as (47), shown at the bottom of the page, where $\boldsymbol{K}_{1}$ and $\boldsymbol{K}_{0}$ represent the subspaces of the possible equivalent transmit vectors $\boldsymbol{K}$ for $b_{k}=1$ and $b_{k}=0$, respectively, and $L_{2, A}\left(b_{j}\right)$ is the a priori LLR of the "outer" decoder corresponding to bits $b_{j}$.
Equations (46) and (47) can be rewritten using the approximate-logarithmic-maximum a posteriori (Approx-logMAP) algorithm [30], [31] as

$$
L_{1, E}\left(b_{k}\right)=\operatorname{jac}_{\boldsymbol{S}_{s}^{q, l} \in \boldsymbol{S}_{1}}\left[d_{1}\right]-\underset{\boldsymbol{S}_{s}^{q, l} \in \boldsymbol{S}_{0}}{\operatorname{jac}}\left[d_{1}\right],
$$

and

$$
L_{2, E}\left(b_{k}\right)=\underset{\boldsymbol{K}_{l, q} \in \boldsymbol{K}_{1}}{\operatorname{jac}}\left[d_{2}\right]-\underset{\boldsymbol{K}_{l, q} \in \boldsymbol{K}_{0}}{\mathrm{jac}}\left[d_{2}\right],
$$

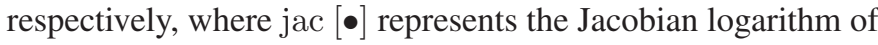
the expression ' $\bullet$ ' and $d_{1}$ and $d_{2}$ are given by

$$
d_{1}=-\frac{\left\|\boldsymbol{z}_{s}^{A}(k)-\bar{h}_{s d}(k) \boldsymbol{S}_{s}^{q, l}\right\|^{2}}{N_{0}}+\sum_{j \neq k} b_{j} L_{1, A}\left(b_{j}\right),
$$

and

$$
\begin{aligned}
d_{2}=-\frac{\left\|\boldsymbol{z}_{v_{1}}^{B}\left(k+L_{f}\right)-\overline{\boldsymbol{H}}_{v_{1} d}\left(k+L_{f}\right) \chi \boldsymbol{K}_{l, q}\right\|^{2}}{N_{0}} & +\sum_{j \neq k} b_{j} L_{2, A}\left(b_{j}\right),
\end{aligned}
$$

respectively. We repeat here that the substitutions detailed in Section IV have to be made for the differential scheme.

Now the exchange of extrinsic information takes place between the DN's demapper-URC-RSC decoder processing frame $n$ (which may be referred to as the "inner" decoder) and the STSK demapper-URC block detecting the VAA frame $(n+1)$ (treated as the "outer" decoder). The extrinsic LLR is appropriately interleaved and deinterleaved by the $\mathrm{SN}$ and by the VAA interleavers and deinterleavers $\Pi_{s}, \Pi_{s}^{-1}, \Pi_{r}$, and $\Pi_{r}^{-1}$, respectively, for the sake of generating the appropriate a priori LLRs for the next iteration. During the last "outer" iteration, the LLR values $L_{1, p}\left(u_{1}\right)$ of the original information bits $u_{1}$ are passed to the hard-decision block of Fig. 5 to estimate the source information. The source information on the next frame is detected in the same manner, processing the frame received directly from the $\mathrm{SN}$ by the $\mathrm{DN}$ and the relayed frame received during the consecutive cooperative frame from the other VAA. This process continues, until the detection of the last frame is completed.

\section{Vi. Performance of the Proposed Scheme}

Here, the performance of our cooperative MC STSK scheme relying on the parameters of Table I is investigated and compared with that of our benchmark schemes. The performance of

$$
L_{2, E}\left(b_{k}\right)=\ln \frac{\sum_{\boldsymbol{K}_{l, q} \in \boldsymbol{K}_{1}} \exp \left[-\frac{\left\|\boldsymbol{z}_{v_{1}}^{B}\left(k+L_{f}\right)-\overline{\boldsymbol{H}}_{v_{1} d}\left(k+L_{f}\right) \boldsymbol{\chi} \boldsymbol{K}_{l, q}\right\|^{2}}{N_{0}}+\sum_{j \neq k} b_{j} L_{2, A}\left(b_{j}\right)\right]}{\sum_{\boldsymbol{K}_{l, q} \in \boldsymbol{K}_{0}} \exp \left[-\frac{\left\|\boldsymbol{z}_{v_{1}}^{B}\left(k+L_{f}\right)-\overline{\boldsymbol{H}}_{v_{1} d}\left(k+L_{f}\right) \boldsymbol{\chi} \boldsymbol{K}_{l, q}\right\|^{2}}{N_{0}}+\sum_{j \neq k} b_{j} L_{2, A}\left(b_{j}\right)\right]}
$$


TABLE I

MAIN Simulation PARAMETERS

\begin{tabular}{|c|c|}
\hline Simulation Parameter & Adopted Value \\
\hline Fast fading model & Correlated Rayleigh fading \\
\hline Doppler frequency & 0.01 \\
\hline Channel specification & $\begin{array}{r}\text { COST 207-TU12, 12-tap channel } \\
\text { delay-spread }=1.0 \mu s[32, \text { Appendix E }]\end{array}$ \\
\hline No. of subcarriers & 64,256 (used only for $S_{f}=256$ ) \\
\hline Length of cyclic prefix & 32 \\
\hline Symbol duration, $T_{s}$ & $500 n s$ \\
\hline CRC code & CRC - 4 \\
\hline Path loss co-efficient, $\alpha$ & \\
\hline No. of RNs ir & \\
\hline No. of Tx time & 2 \\
\hline Distance of VAA fi & $1 / 3$ of SN-DN distance \\
\hline No. of dispersion 1 & $Q=2,4$ \\
\hline STSK specification & $(2,2,2, Q), Q=2,4$ \\
\hline Modulatic & 2,4 \\
\hline Spreading codes & Walsh-Hadamard \\
\hline Spreading factor & $16,64,256$ \\
\hline RSC code & $(2,1,2)$ \\
\hline Generator poly & $\left(g_{r}, g\right)=(3,2)_{8}$ \\
\hline interleavers & $2,400,000$ bits \\
\hline Inner decoding iterations & 2 \\
\hline Outer decoding iterations & 6 \\
\hline
\end{tabular}

the STSK-based scheme, particularly its diversity-multiplexing tradeoff, depends mainly on the specific objective function (OF) used for the optimization of the DMs utilized [1]. More explicitly, the preassigned spreading matrices can be optimized using different OFs, as detailed in [6] and [27]. We have employed an exhaustive search over $10^{6}$ candidate DM sets for minimizing the pairwise symbol error probability under the power constraint as mentioned in Section II-B for the optimization of the DMs used in our proposed scheme. Further detailed discussions on the spreading matrix design can be found in [33]-[35].

Fig. 6 shows the bit-error-rate (BER) performance of the coherent cooperative MC STSK $(2,2,4)$ scheme employing QPSK modulation and compares the performances of different DF schemes in the dispersive typical urban scenario characterized by the COST 207-TU12 channel model. The detailed power and delay profile of the 12 taps that determine the coherence bandwidth and/or delay spread of this channel model may be found in [32] and [36, App. E]. The delay spread of the channel is found to be $\sigma_{\tau}=1.0 \mu \mathrm{s}$, which determines the coherence bandwidth according to [37] $B_{c}=1 /\left(\alpha \cdot \sigma_{\tau}\right)=$ $1 /\left((2 \pi) \cdot \sigma_{\tau}\right) \approx 160 \mathrm{KHz}$, where the value of the constant $\alpha=$ $2 \pi$ is assumed according to [38]. These channel parameters and the overall system's symbol duration of $T_{s}=500 \mathrm{~ns}$ demonstrate that the individual subchannels experience frequency-flat fading, and the length of cyclic prefixes adopted in Table I ensures the absence of ISI. The different DF schemes compared in our investigations, however, are 1) the perfect DF scheme, 2) the proposed scheme assuming perfect interference cancelation, 3) the proposed SR scheme employing CRC-based selective DF, and 4) the conventional DF scheme. The perfect DF scheme represents the proposed scheme, assuming perfect decoding at the RNs, i.e., where all the RNs of each VAA take part in cooperation, whereas the conventional DF schemes allow retransmissions from the VAA RNs without checking whether any decoding error has occurred at the RNs or not. Finally, the perfect-interference-cancelation-oriented scheme assumes that

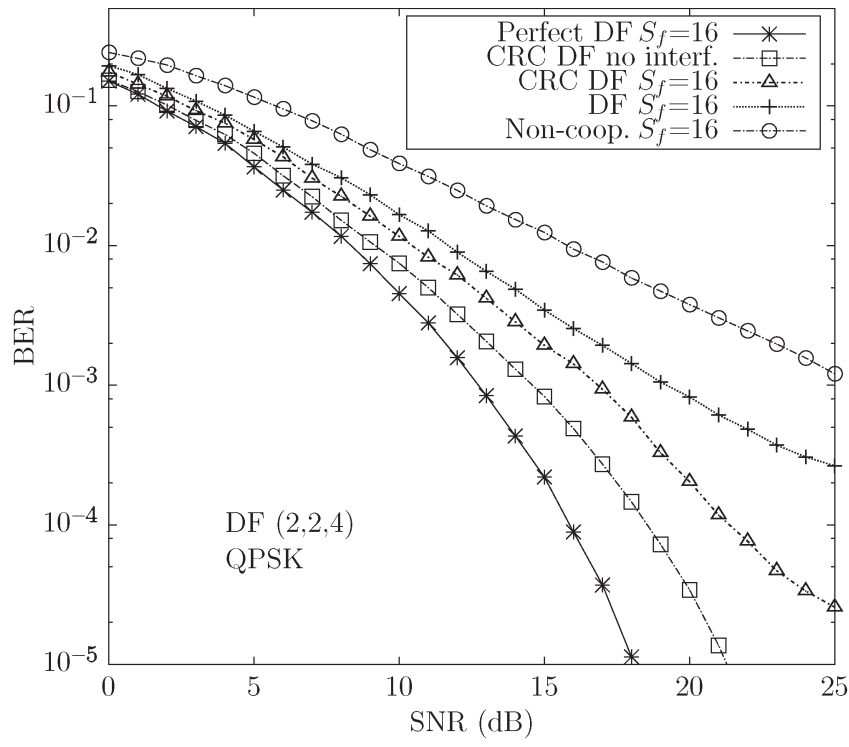

Fig. 6. BER performance of our single-user selective SR MC cooperative coherent STSK $(2,2,4)$ QPSK scheme with $S_{f}=16$ in the dispersive COST207-TU12 channel and other parameters, as shown in Table I, compared against different scenarios, such as the perfect DF scheme, CRC-based scheme assuming perfect interference cancelation, cooperative DF scheme without CRC activation, and the noncooperative QPSK scenario.

no SR-induced interference is imposed. The BER performance of the noncooperative scenario employing QPSK modulation and the same parameters is also shown in Fig. 6. We observe that the proposed CRC-activated scheme can benefit from a higher diversity gain than either the conventional DF or the noncooperative schemes and attains an increased throughput as a benefit of using SR.

To investigate the performance of the interference mitigation process using a double-frame matched filter, the scheme was further studied using spreading codes having different spreading factors. To be specific, the investigations were carried out using the CRC-activated cooperative MC STSK $(2,2,2)$ scheme employing binary PSK (BPSK) modulation relying on $S_{f}=16,64,256$ and the parameters of Table I. The corresponding performance results are presented in Fig. 7. The performance of the proposed scheme is again compared against those of the four different DF schemes as in Fig. 6 and of the noncooperative BPSK scenario. Observe in Fig. 7 that our MC-CDMA-based scheme succeeds in circumventing the channel-induced dispersion and exhibits an improved performance upon increasing the spreading factor. Upon increasing $S_{f}$, the scheme provides additional FD diversity gains, and the specific FD despreading mitigates the SR-induced interference.

The performance of the proposed cooperative MC-CDMA STSK scheme associated with $S_{f}=256$ and recorded for different geographical positions of the VAAs is shown in Fig. 8, together with the achievable multiuser performance. The shapes of the performance curves in single-user scenarios were observed to be shifted toward higher or lower SNRs, owing to the location-related reduced or increased channel gains, respectively. The performance achieved when supporting $U=$ 4 users and an SN-VAA distance of one third relative to the direct SN-DN link is shown in Fig. 8, which is observed to be degraded by MUI. The performance erosion may, however, be 


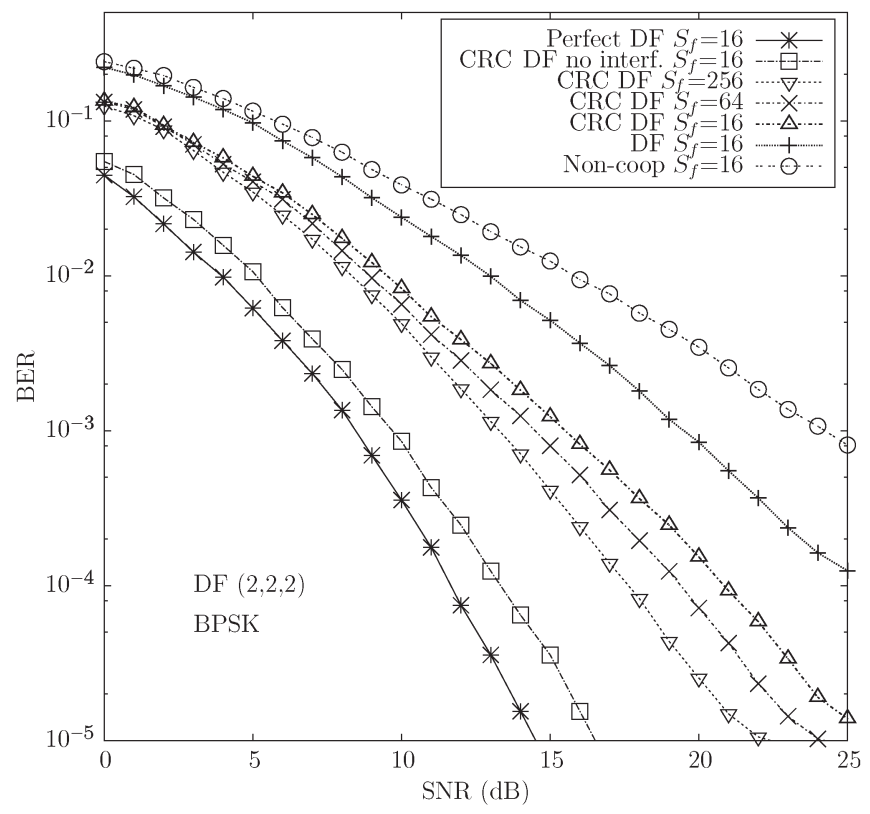

Fig. 7. Achievable BER performance of the proposed $(2,2,2)$ scheme in conjunction with BPSK modulation having $S_{f}=16,64,256$ single users in dispersive COST 207-TU12 channel compared against those with the perfect DF cooperation and with the scheme having complete interference cancelation. The noncooperative benchmarker having the same parameters and the same throughput is also shown.

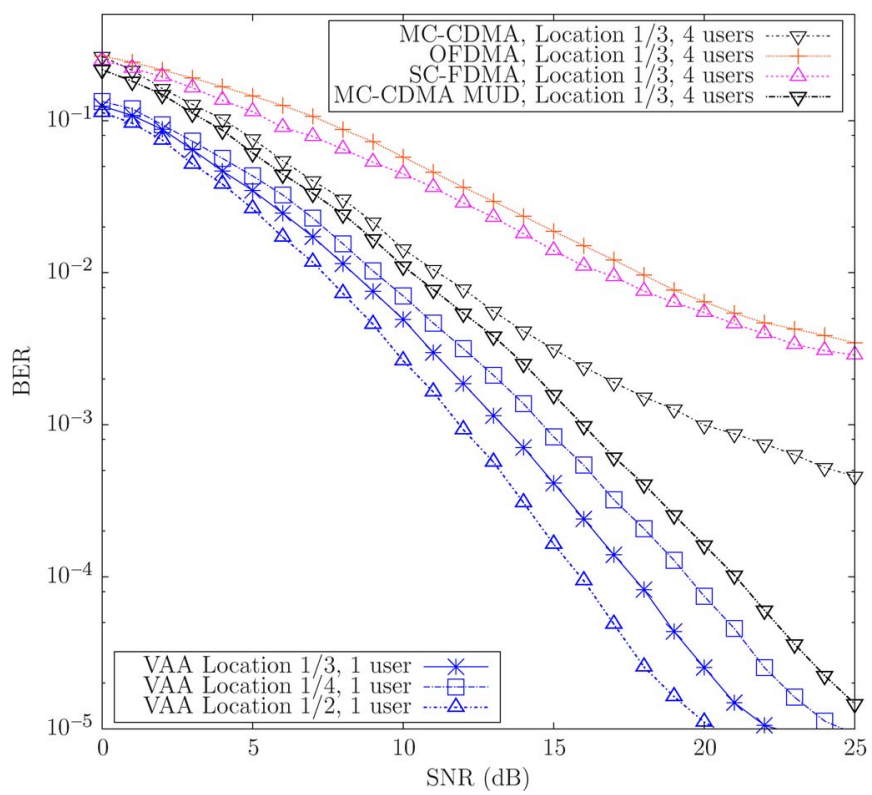

Fig. 8. Performance of the single-user scheme under different SN-VAA distances relative to the direct SN-DN distance. Achievable performance employing different MC systems, namely, MC-CDMA with $S_{f}=256$, OFDMA, SC-FDMA using FD MMSE equalization supporting $U=4$ users, and $1 / 3$ relative SN-VAA distance is also shown, together with the MC-CDMA performance of the ML-MUD, as proposed in (31).

mitigated by employing a MUD formulated in (31). The scheme employing OFDMA and SC-FDMA using FD MMSE equalization and localized subcarrier allocation supporting $U=4$ users, on the other hand, exhibits a further degraded performance, as shown in Fig. 8. This degradation is a consequence of the SRinduced IRI and CCI, demonstrating the benefits of MC-CDMA compared with other candidate MC systems.

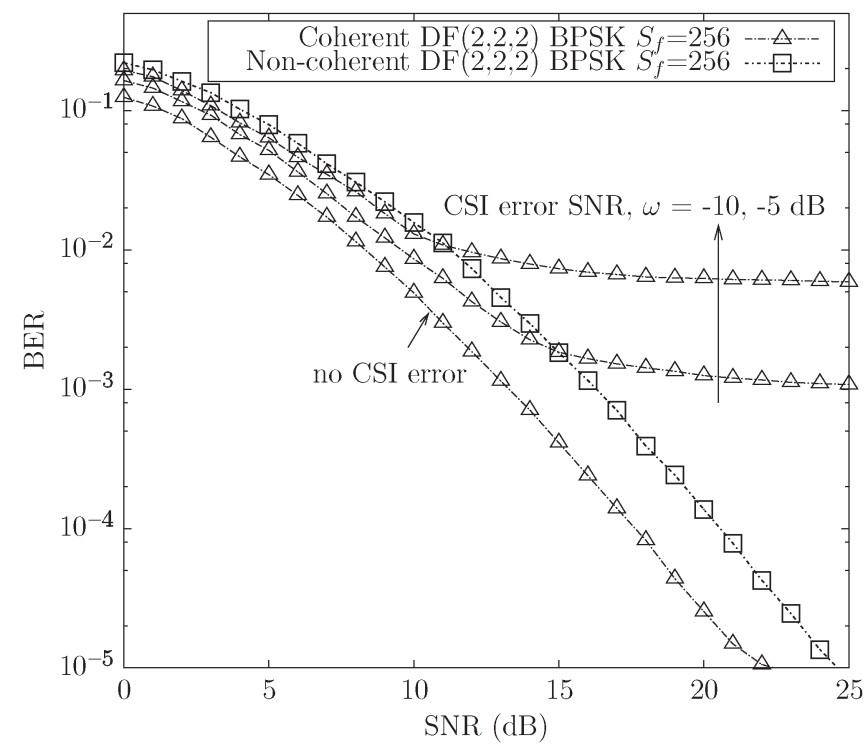

Fig. 9. BER performance of the proposed MC SR BPSK modulated coherent and DSTSK $(2,2,2)$ scheme with $S_{f}=256$ single users in COST 207-TU12 channel. The differential scheme suffers from $-3 \mathrm{~dB}$ performance penalty compared with its coherent counterpart. The effects of the CE errors for the coherent scheme is characterized by the assumed Gaussian CSI estimation error SNR of $\omega=-10$ and $-5 \mathrm{~dB}$.

The performance of our cooperative $\operatorname{DSTSK}(2,2,2)$ scheme relying on BPSK modulation having $S_{f}=256$ is characterized in Fig. 9, which may be directly compared with its coherent counterpart. The effects of the channel state information (CSI) estimation error associated with the coherent scheme are also investigated. More particularly, we assume the CE errors to be Gaussian distributed, and the level of CSI errors is quantified in terms of an equivalent CSI-error SNR of $\omega=-10$ and $-5 \mathrm{~dB}$ below the received signal power. For example, the perfect CSI scenario corresponds to $\omega=-\infty \mathrm{dB}$, whereas $\omega=-10 \mathrm{~dB}$ represents CSI error power, which is one tenth of the received signal power. Observe in Fig. 9 that the differential scheme suffers from a performance penalty of about $3 \mathrm{~dB}$ compared with the perfect-CSI-aided coherent scheme, owing to the inherent noise doubling process of differential encoding. By contrast, the cooperative coherent scheme's performance was severely degraded by the inevitable CSI estimation errors. The FD spreading renders our scheme less susceptible to CSI errors because a bit might still become recoverable if some of the spreading-code chips become corrupted. Nonetheless, the coherent scheme is seen to exhibit a considerable error floor in Fig. 9. Moreover, the coherent scheme requires the transmission of pilot symbols, in addition to the CRC overhead. In the light of the impediments of the coherent scheme mentioned previously, the differential MC STSK system may be deemed an attractive candidate for cooperative MIMO-aided MC communications.

Fig. 10 characterizes the achievable BER performance of the soft-decision-aided channel-coded cooperative MC-CDMA STSK $(2,2,4)$ QPSK scheme using $S_{f}=16$ in the context of wideband channels, where we have employed a half-rate RSC code having a constraint length of $k=2$, the generator polynomials of $\left(g_{r}, g\right)=(3,2)_{8}$, and two random interleavers of length 2.4 million bits. Both the coherent and the differential cooperative schemes are benchmarked against the 


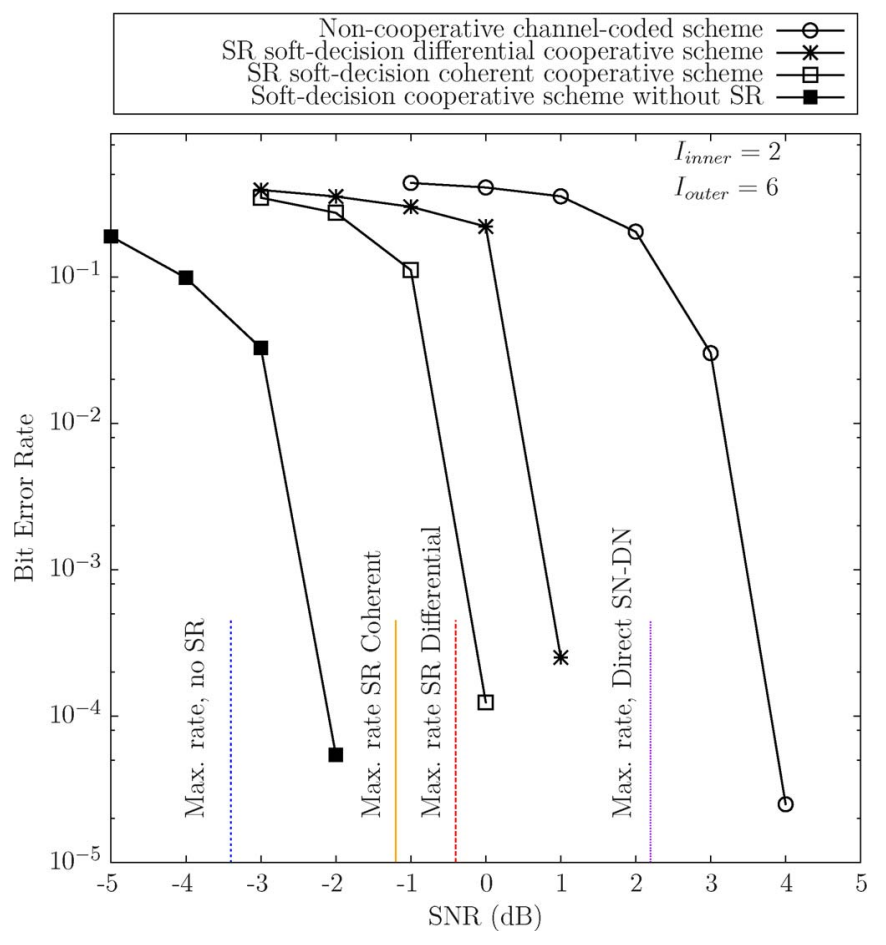

Fig. 10. Achievable performance of soft-decision three-stage turbo cooperative MC-CDMA STSK $(2,2,4)$ QPSK with $S_{f}=16$ single users communicating over the COST207-TU12 channel $\left(f_{d}=0.01\right)$. The performance of both the coherent and differentially encoded SR schemes is provided with that of the direct noncooperative and half-duplex cooperative benchmarkers. The maximum achievable rates of the corresponding schemes, computed by the EXIT-chart-based analysis, are also provided.

noncooperative scheme and against the cooperative arrangement employing no SR schemes. As observed in Fig. 10, the noncoherent scheme exhibits a slight performance degradation compared with its coherent counterpart. However, the noncoherent scheme has the potential advantage of dispensing with CE. The noncooperative scheme exhibits a substantially eroded performance, whereas the half-duplex scheme shows a somewhat better performance, albeit this is achieved at the cost of a severe throughput loss. The number of inner and outer decoder iterations was set to $I_{\text {inner }}=2$ and $I_{\text {outer }}=6$, respectively. The maximum achievable rates were estimated by evaluating the area under the EXIT chart of the corresponding inner decoder, which are shown in Fig. 10. To be more specific, we exploited using the area property of EXIT charts, as discussed in [39] and [40], which states that the maximum achievable rate is determined by the area under the inner decoder's EXIT curve, whereas the maximum capacity $C_{\max }$ may be formulated as

$$
C_{\text {max }}(\mathrm{SNR}) \approx R \cdot A_{\text {inner }}(\mathrm{SNR})
$$

where $A_{\text {inner }}$ is the aforementioned area corresponding to a certain SNR value, and $R$ is the number of bits per symbol.

Fig. 11 shows the EXIT chart of the SR-aided cooperative MC-CDMA STSK $(2,2,4)$ QPSK scheme using $S_{f}=16$ at a channel SNR of $0 \mathrm{~dB}$. It is shown in Fig. 11 that the inner decoder's EXIT curve reached the point of perfect decoding convergence $(1.0,1.0)$, which is the explicit benefit of employing URC precoding [27]. We also observe that an open EXIT tunnel was formed at $\mathrm{SNR}=0 \mathrm{~dB}$, and the EXIT curve at $\mathrm{SNR}=$ $0 \mathrm{~dB}$ was also confirmed by the corresponding Monte Carlo-

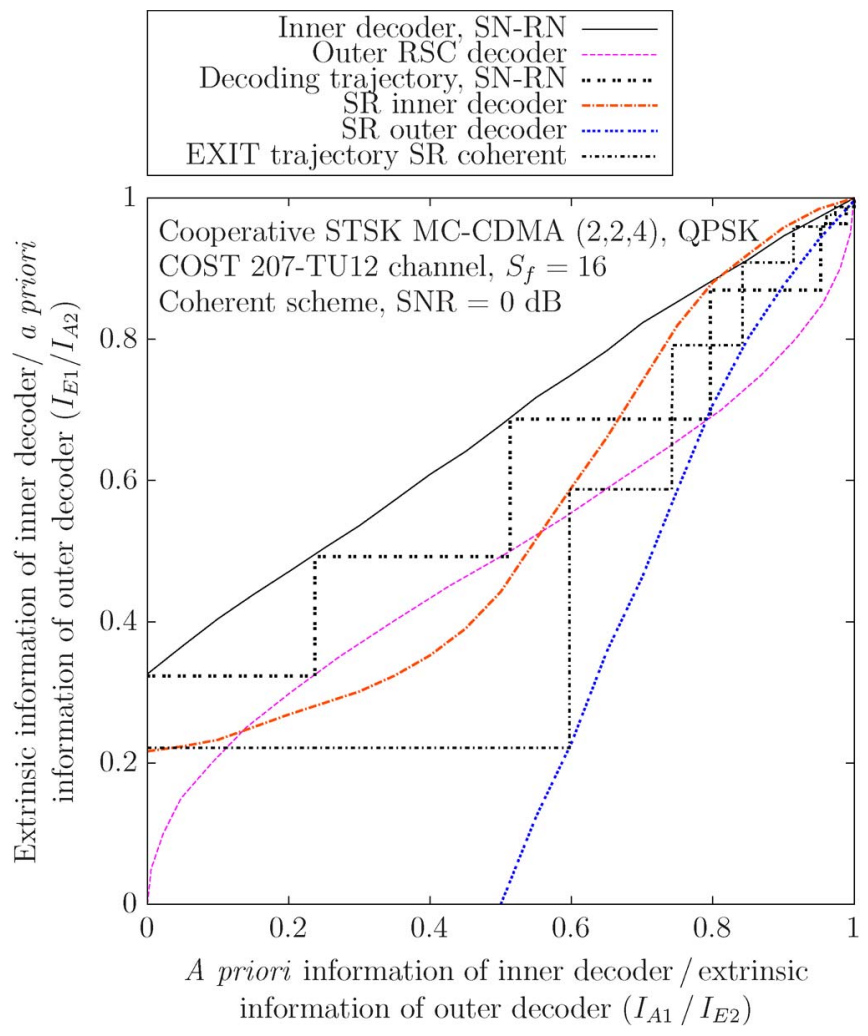

Fig. 11. EXIT trajectory recorded at $0 \mathrm{~dB}$ of our three-stage turbo detected SR-aided cooperative MC-CDMA STSK $(2,2,4)$ QPSK with $S_{f}=16$ single users communicating over the COST207-TU12 channel $\left(f_{d}=0.01\right)$, together with the inner decoder EXIT curves at $0 \mathrm{~dB}$ and the outer decoder EXIT function.

simulation-based staircase-shaped decoding trajectory [41]. Therefore, it may be predicted that an infinitesimally low BER is achieved at $\mathrm{SNR}=0 \mathrm{~dB}$ using $I_{\text {outer }}=6$ outer iterations.

\section{CONCLUSION}

In this paper, we proposed a novel cooperative MC STSK scheme using selective DF and SR to recover the half-duplex multiplexing loss. The scheme is capable of striking a flexible diversity versus multiplexing gain tradeoff with the aid of the recent STSK concept at low decoding complexity.

The SR regime assists in recovering the half-duplex throughput loss at the cost of imposing inter-VAA interference and interstream interference at the DN [15], [16]. The problem of Inter-VAA interference is eliminated by invoking the proposed CRC-based selective DF cooperation along with the specific FD despreading regime used, whereas the interstream interference is mitigated by using our double-frame-based chip-waveform matched filter [17] along with the proposed joint single-streambased ML decoding.

Furthermore, to overcome the performance degradation imposed by CE errors, we proposed a cooperative MC DSTSK scheme, which retained all the fundamental benefits of the coherent scheme. As a further advance, we also proposed a serially concatenated channel-coded and soft-decision-based iteratively decoded cooperative STSK architecture. In a nutshell, the scheme has the inherent design flexibility of adaptively selecting the number of RNs in the VAAs and the ability to strike a flexible rate-diversity tradeoff, depending 
on the near-instantaneous channel conditions while providing protection against the frequency selectivity of the channel.

\section{REFERENCES}

[1] S. Sugiura, S. Chen, and L. Hanzo, "A universal space-time architecture for multiple-antenna aided systems," IEEE Commun. Surveys Tuts., vol. 14, no. 2, pp. 401-420, Second Quart., 2012.

[2] S. Sugiura, S. Chen, and L. Hanzo, "Coherent and differential space-time shift keying: A dispersion matrix approach," IEEE Trans. Commun., vol. 58, no. 11 , pp. 3219-3230, Nov. 2010.

[3] S. Sugiura, S. Chen, and L. Hanzo, "Generalized space-time shift keying designed for flexible diversity-, multiplexing- and complexity-tradeoffs," IEEE Trans. Wireless Commun., vol. 10, no. 4, pp. 1144-1153, Apr. 2011

[4] R. Mesleh, H. Haas, S. Sinanovic, C. W. Ahn, and S. Yun, "Spatial modulation," IEEE Trans. Veh. Technol., vol. 57, no. 4, pp. 2228-2241, Jul. 2008.

[5] B. Hassibi and B. M. Hochwald, "High-rate codes that are linear in space and time," IEEE Trans. Inf. Theory, vol. 48, no. 7, pp. 1804-1824, Jul. 2002.

[6] R. W. Heath, Jr. and A. Paulraj, "Linear dispersion codes for MIMO systems based on frame theory," IEEE Trans. Signal Process., vol. 50, no. 10 , pp. 2429-2441, Oct. 2002.

[7] V. Tarokh, H. Jafarkhani, and A. Calderbank, "Space-time block codes from orthogonal designs," IEEE Trans. Inf. Theory, vol. 45, no. 5, pp. 1456-1467, Jul. 1999.

[8] C. J. Foschini, "Layered space-time architecture for wireless communication in a fading environment when using multiple antennas," Bell Labs. Tech. J., vol. 1, no. 2, pp. 41-59, 1996.

[9] P. Wolniansky, G. Foschini, G. Golden, and R. Valenzuela, "V-BLAST: An architecture for realizing very high data rates over the rich-scattering wireless channel," in Proc. ISSSE, Sep./Oct. 1998, pp. 295-300.

[10] G. Foschini, G. Golden, R. Valenzuela, and P. Wolniansky, "Simplified processing for high spectral efficiency wireless communication employing multi-element arrays," IEEE J. Sel. Areas Commun., vol. 17, no. 11, pp. 1841-1852, Nov. 1999.

[11] S. Sugiura, C. Xu, S. X. Ng, and L. Hanzo, "Reduced-complexity coherent versus non-coherent QAM-aided space-time shift keying," IEEE Trans. Commun., vol. 59, no. 11, pp. 3090-3101, Nov. 2011.

[12] J. Laneman and G. Wornell, "Distributed space-time-coded protocols for exploiting cooperative diversity in wireless networks," IEEE Trans. Inf. Theory, vol. 49, no. 10, pp. 2415-2425, Oct. 2003.

[13] T. Wang, A. Cano, G. Giannakis, and J. Laneman, "High-performance cooperative demodulation with decode-and-forward relays," IEEE Trans. Commun., vol. 55, no. 7, pp. 1427-1438, Jul. 2007.

[14] S. Sugiura, S. Chen, H. Haas, P. Grant, and L. Hanzo, "Coherent versus non-coherent decode-and-forward relaying aided cooperative space-time shift keying," IEEE Trans. Commun., vol. 59, no. 6, pp. 1707-1719, Jun. 2011.

[15] Y. Fan, C. Wang, J. Thompson, and H. Poor, "Recovering multiplexing loss through successive relaying using repetition coding," IEEE Trans. Wireless Commun., vol. 6, no. 12, pp. 4484-4493, Dec. 2007.

[16] L. Kong, S. X. Ng, R. Maunder, and L. Hanzo, "Near-capacity cooperative space-time coding employing irregular design and successive relaying," IEEE Trans. Commun., vol. 58, no. 8, pp. 2232-2241, Aug. 2010.

[17] L. Li and L. Hanzo, "Multiple-symbol differential sphere detection aided successive relaying in the cooperative DS-CDMA uplink," in Proc. IEEE WCNC, Mar. 2011, pp. 1875-1880.

[18] L. Li, L. Wang, and L. Hanzo, "Successive AF/DF relaying in the cooperative DS-CDMA uplink: Capacity analysis and its system architecture," IEEE Trans. Veh. Technol., vol. 62, no. 2, pp. 655-666, Feb. 2013.

[19] P. Zhang, S. Chen, and L. Hanzo, "Differential space-time shift keying aided successive relaying assisted cooperative multi-user CDMA," IEEE Trans. Veh. Technol., vol. 62, no. 5, pp. 2156-2169, Jun. 2013.

[20] M. I. Kadir, S. Sugiura, J. Zhang, S. Chen, and L. Hanzo, "OFDMA/ SC-FDMA aided space-time shift keying for dispersive multiuser scenarios," IEEE Trans. Veh. Technol., vol. 62, no. 1, pp. 408-414, Jan. 2013.

[21] M. Driusso, F. Babich, M. I. Kadir, and L. Hanzo, "OFDM aided space-time shift keying for dispersive downlink channels," in Proc. IEEE VTC Fall, Sep. 2012, pp. 1-5.

[22] J. Jeganathan, A. Ghrayeb, and L. Szczecinski, "Spatial modulation: Optimal detection and performance analysis," IEEE Commun. Lett., vol. 12, no. 8, pp. 545-547, Aug. 2008.

[23] C. Xu, S. Sugiura, S. X. Ng, and L. Hanzo, "Reduced-complexity noncoherently detected differential space-time shift keying," IEEE Signal Process. Lett., vol. 18, no. 3, pp. 153-156, Mar. 2011.
[24] L. Hanzo, M. Munster, B. J. Choi, and T. Keller, OFDM and MC-CDMA for Broadcasting Multi-User Communications, WLANs and Broadcasting. New York, NY, USA: Wiley, 2003.

[25] J. Laneman, D. Tse, and G. Wornell, "Cooperative diversity in wireless networks: Efficient protocols and outage behavior," IEEE Trans. Inf. Theory, vol. 50, no. 12, pp. 3062-3080, Dec. 2004.

[26] S. Hara and R. Prasad, "Overview of multi-carrier CDMA," IEEE Commun. Mag., vol. 35, no. 12, pp. 126-133, Dec. 1997.

[27] L. Hanzo, O. Alamri, M. El-Hajjar, and N. Wu, Near-Capacity MultiFunctional MIMO Systems (Sphere-Packing, Iterative Detection and Cooperation). New York, NY, USA: Wiley, 2009.

[28] S. Verdú, Multiuser Detection. New York, NY, USA: Cambridge Univ. Press, 1998

[29] S. ten Brink, J. Speidel, and R.-H. Yan, "Iterative demapping and decoding for multilevel modulation," in Proc. IEEE GLOBECOM, 1998, vol. 1, pp. $579-584$.

[30] L. Hanzo, T. Liew, and B. Yeap, Turbo Coding, Turbo Equalisation and Space-time Coding for Transmission over Fading Channels. New York, NY, USA: Wiley, 2002.

[31] J. Woodard and L. Hanzo, "Comparative study of turbo decoding techniques: An overview," IEEE Trans. Veh. Technol., vol. 49, no. 6, pp. 2208 2233, Nov. 2000.

[32] "Digital land mobile radio communications," Office Official Pub. Eur. Commun., Luxembourg, Tech. Rep. COST 207, 1989.

[33] M. Jiang and L. Hanzo, "Unitary linear dispersion code design and optimization for MIMO communication systems," IEEE Signal Process. Lett., vol. 17, no. 5, pp. 497-500, May 2010.

[34] F. Babich, A. Crismani, M. Driusso, and L. Hanzo, "Design criteria and genetic algorithm aided optimization of three-stage-concatenated space-time shift keying systems," IEEE Signal Process. Lett., vol. 19, no. 8, pp. 543-546, Aug. 2012.

[35] R. Rajashekar, K. Hari, and L. Hanzo, "Field extension code based dispersion matrices for coherently detected space-time shift keying," in Proc. IEEE GLOBECOM, Dec. 2011, pp. 1-5.

[36] M. Patzold, Mobile Fading Channels. New York, NY, USA: Wiley, 2003.

[37] M. Gans, "A power-spectral theory of propagation in the mobile-radio environment," IEEE Trans. Veh. Technol., vol. VT-21, no. 1, pp. 27-38, Feb 1972.

[38] W. C. Jakes and D. C. Cox, Eds., Microwave Mobile Communications. New York, NY, USA: Wiley-IEEE Press, 1994.

[39] M. Tuchler, "Design of serially concatenated systems depending on the block length," IEEE Trans. Commun., vol. 52, no. 2, pp. 209-218, Feb. 2004.

[40] A. Ashikhmin, G. Kramer, and S. ten Brink, "Extrinsic information transfer functions: Model and erasure channel properties," IEEE Trans. Inf. Theory, vol. 50, no. 11, pp. 2657-2673, Nov. 2004.

[41] S. ten Brink, "Convergence behavior of iteratively decoded parallel concatenated codes," IEEE Trans. Commun., vol. 49, no. 10, pp. 1727-1737, Oct. 2001.

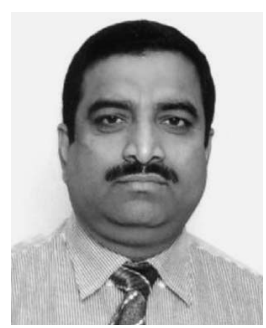

Mohammad Ismat Kadir received the B.Sc. Eng. degree (with first-class) in electrical and electronic engineering and the M.Sc. Eng. degree in computer engineering from Bangladesh University of Engineering and Technology, Dhaka, Bangladesh, in 1992 and 1999, respectively. He is currrently working toward the $\mathrm{Ph} . \mathrm{D}$. degree with the Commmunications, Signal Processing, and Control Group, School of Electronics and Computer Science, University of Southampton, Southampton, U.K.

His research interests include multicarrier systems, space-time coding, cooperative communications, error control coding, and iterative detection.

Mr. Kadir received the Commonwealth Scholarship from the Commonwealth Scholarship Commission, U.K. 


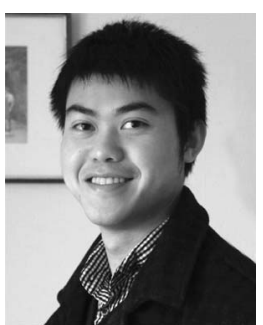

Li Li received the B.Eng. degree in information engineering from the University of Electronic Science and Technology of China, Chengdu, China, in 2006 and the M.Sc. degree, with distinction, in wireless communications from the University of Southampton, Southampton, U.K., in 2009, where he is currently working toward the Ph.D. degree with the Communications Group, School of Electronics and Computer Science.

$\mathrm{He}$ is currently participating in the European Union Concerto Project. His research interests include channel coding, iterative detection, noncoherent transmission technologies, cooperative communications, and network coding.

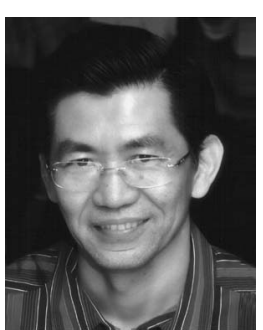

Sheng Chen (M'90-SM'97-F'08) received the B.Eng. degree in control engineering from the East China Petroleum Institute, Beijing, China, in 1982; the Ph.D. degree in control engineering from City University London, London, U.K., in 1986; and the D.Sc. degree from the University of Southampton, Southampton, U.K., in 2005.

From 1986 to 1999, he held research and academic appointments with the University of Sheffield, Sheffield, U.K.; the University of Edinburgh, Edinburgh, U.K.; and the University of Portsmouth, Portsmouth, U.K. He is a Distinguished Adjunct Professor with King Abdulaziz University, Jeddah, Saudi Arabia. Since 1999, he has been with the School of Electronics and Computer Science, the University of Southampton, where he is currently a Professor of intelligent systems and signal processing. He is the author of 460 research papers. He was an Institute for Scientific Information Highly Cited Researcher in the engineering category in March 2004. His research interests include adaptive signal processing, wireless communications, modeling and identification of nonlinear systems, neural networks and machine learning, intelligent control system design, and evolutionary computation methods and optimization.

Dr. Chen is a Fellow of The Institution of Engineering and Technology.

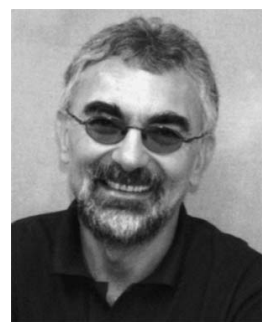

Lajos Hanzo (M'91-SM'92-F'04) received the M.S. degree (with first-class honors) in electronics and the Ph.D. degree from the Technical University of Budapest, Budapest. Hungary, in 1976 and 1983 , respectively, the D.Sc. degree from the University of Southampton, Southampton, U.K., in 2004, and the "Doctor Honoris Causa" degree from the Technical University of Budapest in 2009.

During his 35-year career in telecommunications, he has held various research and academic posts in Hungary, Germany, and the U.K. Since 1986, he has been with the School of Electronics and Computer Science, University of Southampton, where he holds the Chair in Telecommunications. Since 2009, he has been a Chaired Professor with Tsinghua University, Beijing, China. $\mathrm{He}$ is currently directing a 100 -strong academic research team, working on a range of research projects in the field of wireless multimedia communications sponsored by industry; the Engineering and Physical Sciences Research Council, U.K.; the European Information Society Technologies Program; and the Mobile Virtual Centre of Excellence, U.K. He is an enthusiastic supporter of industrial and academic liaison and offers a range of industrial courses. $\mathrm{He}$ has successfully supervised $80 \mathrm{Ph}$.D. students, coauthored 20 JohnWiley/IEEE Press books on mobile radio communications totaling in excess of 10000 pages, published more than 1250 research entries on IEEE Xplore, and presented keynote lectures. For further information on research in progress and associated publications, see http://www-mobile.ecs.soton.ac.uk.

Dr. Hanzo is a Fellow of the Royal Academy of Engineering, U.K., a Fellow of the Institution of Electrical Engineers, and a Governor of the IEEE Vehicular Technology Society. He has been a Technical Program Committee Chair and a General Chair for IEEE conferences. During 2008-2012, he was the Editor-inChief of the IEEE Press. He has received a number of distinctions. 